\title{
Role of Optimal Screening and Treatment on Infectious Diseases Dynamics in Presence of Self-protection of Susceptible
}

\author{
Anuj Kumar ${ }^{1,2} \cdot$ Prashant K. Srivastava $^{2}$ (D) \\ Published online: 27 March 2019 \\ (c) Foundation for Scientific Research and Technological Innovation 2019
}

\begin{abstract}
In this study, a nonlinear SEIR model has been proposed and analysed that accounts for the screening of exposed population, limited treatment of infective and self-protection in susceptible population. We observe that for the basic reproduction number $\left(\mathcal{R}_{0}\right)$ below one, a backward bifurcation exists due to saturation in treatment. Also, $\mathcal{R}_{0}$ depends on selfprotection parameter and hence self-protection can help in reducing it. Further to understand the impact of screening and treatment on disease dynamics, we extend this model to an optimal control problem. Using Pontryagin's Maximum Principle, optimal control paths are obtained analytically corresponding to the proposed cost functional. Our numerical experimentations suggest that between only screening and only treatment control policies, screening is more effective and economically viable than the only treatment policy. The combined usage of both screening and treatment is found highly effective and least expensive. Hence, we conclude that implementation of screening along with treatment not only reduces the infection level but also minimizes the associated economic burden. Also, when optimal controls are applied, the cumulative count of infective reduces with the increase in self-protection, therefore, selfprotection helps in reducing the disease burden. The epidemic peak as well as cost is also shown to be reduced when self-protection is high for various values of basic reproduction number.
\end{abstract}

Keywords Global stability · Backward bifurcation · Self-protection · Screening · Limited treatment $\cdot$ Optimal control

\section{Introduction}

It is well recognized that disease outbreaks seriously affect the economy of communities due to high morbidity and mortality as well as various disease-related costs (such as expenditures

Prashant K. Srivastava

pksri@iitp.ac.in

Anuj Kumar

anujdubey17@gmail.com

1 School of Mathematics, Thapar Institute of Engineering and Technology, Patiala 147004, India

2 Department of Mathematics, Indian Institute of Technology Patna, Patna 801103, India 
on health care, diagnosis and medical treatment etc. as well as productivity loss due to morbidity and mortality) $[1,3,24]$. Infectious diseases like influenza, flu, SARS etc. are some major contributors of these issues globally among many others $[1,3]$. Thus, the control and prevention of disease transmission have become utmost essential.

There are many challenges in detection, treatment, and control of infective. Detection of infected individuals in asymptomatic phase is rather difficult, challenging and expensive in comparison to those showing symptoms [36]. But control of such individuals is essential as they belong to high-risk group and will be infectious in future. Once identified, the treatment can be carried out to reduce mortality and morbidity. Also, it has been observed that when a disease outbreaks in a population, healthy individuals tend to change their behaviour by adopting protective measures to avoid contracting infection $[4,45]$. In this work, we propose to study the impact of these three major interventions: screening, treatment and behavioural change on the dynamics of disease using a mathematical and optimal control model.

Mathematical models have been used extensively to predict, control and also to formulate policies to eradicate or contain diseases including the interventions mentioned in the previous paragraph $[5,8,19,25,27,28,30,38,43,44,50]$. For example, screening or identification on active infectious population has been used by many researchers as an intervention [5,23,30]. Lee et al. [36] in 1988 modeled the impact of mass screening programs on the dynamics of contagious and non-contagious diseases. In 2004, Gumel et al. [23] formulated and studied an epidemic model for SARS outbreaks implementing two important strategies: one- the quarantine of asymptomatic (exposed) individuals and, second- the isolation of infectious individuals. They showed that their model pattern resembles the pattern of the data collected during outbreaks in GTA, Hong Kong, Singapore, and Beijing. They also noted that isolation is an important strategy in controlling the outbreak of SARS and eradication is feasible with optimal isolation along with highly effective screening. Various mitigation strategies, other than screening, such as isolation, quarantine etc. have also been studied to control the spread of SARS, HIV and other sexually transmitted diseases [5,6,23,29,30,36,44,49].

Control strategies such as vaccination, treatment, screening, quarantine, isolation, educational campaigns and self-protective mechanism along with their cost determination have been studied by various researchers $[5,7,10,19,27,28,31,49,50]$. There is always a trade-off in the execution of suitable control strategies and its cost minimization. In 2010, study for pandemic influenza using antiviral treatment and isolation as control measures was performed by Lee et al. [37]. Authors designed and analysed five control strategies using various combinations of antiviral treatment and isolation that are under resource restrictions. They found that integrated strategy reduces maximum pandemic peak whereas isolation works well when antiviral resources are limited [37]. Armbruster et al. [5,6] used two mitigation policies, such as screening and contact tracing, to control the spread of infectious diseases and formulated control problem with aim to find the cheapest mix policy of screening and contact tracing which minimizes disease prevalence. Yan et al. [49] extended the model of Gumel et al. [23] to corresponding optimal control problem considering quarantine and isolation as control measures for SARS epidemic. In 2013, Okosun et al. [44] studied the optimal impact of treatment for HIV patients and screening of unaware infective in which force of infection consists of the different level of infection. Further authors carried out the cost-effective analysis for proposed optimal control model and found that combined strategy of treatment and screening is more reliable and cost-effective during the outbreaks. In 2000, vaccination, quarantine, screening, and health promotional campaigns were implemented as control measures by Behncke [7] for an epidemic model and he found that the impact of screening on infective is very effective. He also explained the role of the economical asset in promoting the health campaigns and their effect on disease progression via optimal control. Castilho [10], in 2006, 
proposed a model of epidemic control through educational campaigns and described that such control strategies reduce maximum infective load under the limited budget. In 2009, Gaff et al. [19] formulated epidemiological models using vaccination and treatment as control measures. They observed that comprehensive use of vaccination and treatment plays a critical role in the reduction of disease burden, whereas, vaccination only strategy is found more effective than treatment only strategy. Zeiler et al. [50] in 2010 formulated a model in which force of infection is modified through behavioural change and further considered an optimal control problem with screening policy for infective. Recently in 2015, Kassa et al. [28] proposed and studied the impact of self-protective measures via education on an optimal control problem using various control policies. They found that optimal treatment along with behavioural changes through education plays a central role in the dynamics of diseases.

It is observed that information about disease prevalence influences the behaviour of healthy individuals and they adopt protective measures such as use of masks, sanitizer and condoms etc. $[4,9,14,15,18,28,40]$ to abate the disease transmission. Alberto d'Onofrio and his co-workers $[9,14,15]$ accounted for the effect of information-related change in contact pattern and in vaccination process. They observed that delayed information and information-dependent vaccination coverage cause the oscillations when endemic equilibrium changes their stability and also established the global stability of equilibrium conditionally. They numerically validated the obtained results considering various forms of information-depended vaccine coverage. Liu et al. [40] in 2008 proposed and analysed an SIRS model where the force of infection was modified through media effect due to the prevalence of disease and found that awareness reduces the epidemic peaks. The effect of awareness/information rise due to disease prevalence in the population is modeled by Funk et al. [18] and they studied and explained that the change in human behaviour due to information reduces the infectivity. Some other similar work can also be found in $([22,42,48]$ and references therein).

Infection in populations occurs via interaction of infective with susceptible. Bilinear interaction is one of the common forms used for interaction. It is density dependent and leads to unbounded interaction $[8,25]$. However, due to crowding effect, a saturation effect is observed which leads to nonlinear interaction. In fact, the psychological effect and/or behavioural changes of healthy individuals also may be reflected in nonlinear responses. These behavioural changes induce inhibitory effect that reduces the force of infection (see [16,47,51-53] and references therein). In [16,47,53], authors observed different kinds of biological phenomena such as backward bifurcation, global behaviour of infected equilibrium, oscillatory behaviour of infected population and few other aspects using different kinds of force of infection function. This is caused due to saturation in treatment rate which forces infected individuals to be queued up for treatment as medical resources are limited.

In this work, we assume that information about disease prevalence and protective measures are available in population. We quantify the effect of information on susceptible individuals so that they adopt suitable protective measures i.e. 'self-protection' to abate transmission influenced by information about disease prevalence. Since these individuals are using protective measures, we assume that they become virtually immune to the infection and hence move to recovered class. Further, as we know exposed class is highly risky for disease prevalence and will be infectious soon, we incorporate the effect of screening that includes diagnosis along with cure of exposed individuals. Such screening may be provided to the high risk groups or to region where the prevalence of disease is higher. A similar type of policy for screening has been found in practice in control of STDs in India [2]. To treat the infective individuals so as to reduce their prevalence in population during the course of the epidemic, we assume the treatment is available. As the medical resources are limited we consider sat- 
urated treatment on infective. Our aim in this work is to study the effect of these aspects (self-protection, screening, and limited treatment) on the dynamics of the disease and how these can be used to reduce the load of infective. Further, using screening and treatment as control interventions, we intend to obtain optimal policy that not only reduced the disease load but also minimizes the cost so incurred.

The structure of the rest paper is as follows: in the following section, we formulate a mathematical model which accounts for the effect of self-protection on susceptible individuals, screening effort on exposed individuals and saturated treatment on active infectious individuals. In the next section, we study the "Equilibrium Analysis" and "Bifurcation Analysis". Further, we formulate the corresponding "Optimal Control Problem" and analyse it in the following section. We numerically simulate models and validate analytical results with the discussion in the next section. The observations and results are given in final section.

\section{Mathematical Model}

In this section, we formulate a nonlinear compartmental model (SEIR) which deals with the dynamics of infection where healthy individuals have information about disease prevalence. Consider that the total population $N$, is divided in the following compartments: $S$-susceptible population (who are prone to get infection from infective), $E$-exposed population (those individuals who have infection but not showing symptoms and also not able to spread infection), $I$-infective population (those who have the infection and capable to infect other individuals actively), and $R$-recovered population (those who are immune to infection after getting treated or using self-protection). We also assume that the asymptomatic individuals are noninfectious and they coincide with the exposed cohort.

As it has been observed that in the presence of disease, susceptible individuals protect themselves by adopting suitable protective measures (i.e. self-protection) such as the use of masks, sanitizer, vaccination, condoms etc. which provides 'virtual immunity' [4,28,41]. We consider that a fraction of such individuals $\left(h_{1}(S)=\theta S\right)$ will remain protected from infection and therefore they will be virtually immune to the infection and hence will move to $R$ class. Parameter $\theta$ is self-protection rate from the susceptible to the removed class via taking available protective measures. Further, the effect of screening on exposed individuals (that includes diagnosis along with cure of exposed individuals) and saturated treatment of infective have also been incorporated to control the disease progression and prevalence. The corresponding schematic diagram of the model dynamics is given in Fig. 1.

The model dynamics is described by following system of nonlinear ODEs:

$$
\begin{aligned}
\frac{d S}{d t} & =\Lambda-\frac{\beta S I}{1+m I}-\mu S-h_{1}(S), \\
\frac{d E}{d t} & =\frac{\beta S I}{1+m I}-(\mu+\epsilon) E-h_{2}(E), \\
\frac{d I}{d t} & =\epsilon E-(\mu+d+\gamma) I-h_{3}(I), \\
\frac{d R}{d t} & =\gamma I-\mu R+h_{1}(S)+h_{2}(E)+h_{3}(I),
\end{aligned}
$$

with initial conditions $S(0)>0, E(0) \geq 0, I(0) \geq 0$ and $R(0) \geq 0$.

Here, all the parameters are non-negative. The parameter $\Lambda$ represents the growth rate of the susceptible population, $\gamma$ is per capita constant recovery rate of the infected population 


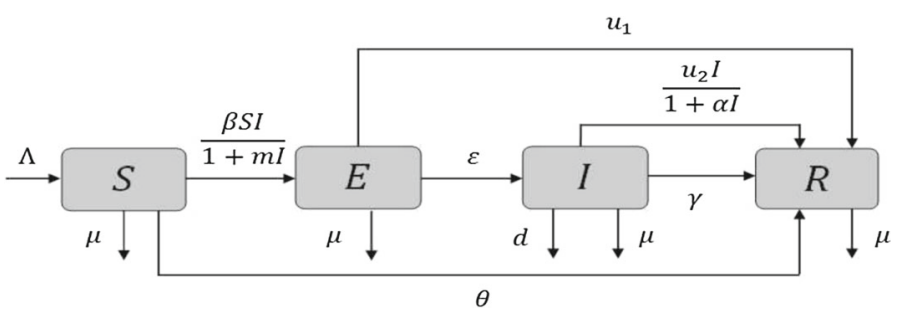

Fig. 1 Schematic diagram of disease dynamics under effect of screening and treatment along with selfprotection

due to natural immunity, $\mu$ is per capita natural mortality rate and assume to be same for each compartment, and $d$ is the corresponding disease related death rate. $\beta$ is transmission rate of the disease and the factor $\frac{\beta S I}{1+m I}$ represents the saturated type of force of infection where $m$ is nonnegative constant related with saturation in transmission. This saturated type force of infection describes that if the count of the infective is at a low level then this works like standard bilinear transmission whereas later due to crowding of infective this transmission reaches to a saturation level. The per capita growth rate of infective is $\epsilon$ when exposed individuals become infective. The term $h_{2}(E)$ denotes the screening rate of the exposed population. We assume that the screening is implemented linearly in the population. Hence, we consider this rate as $h_{2}(E)=u_{1} E$, where $u_{1}$ represents the per capita screening rate implemented on the exposed class. We justify choosing linear function by arguing that the screening should be applied to high risk groups which can be easily identified. Further, this also helps in keeping model equations simple. The factor $h_{3}(I)$ represents the treatment implemented to the infective population. We consider a saturated or limited treatment as $h_{3}(I)=\frac{u_{2} I}{1+\alpha I}$ due to limitations on medical facilities. Here, $\alpha$ is nonnegative constant related to saturation in treatment and $u_{2}$ is treatment rate of infective individuals.

\section{Positivity and Boundedness of Solutions}

Here, we shall show that if we start with positive initial populations, all future populations will remain positive for all time. From the model system (1), with the functions $h_{i}, i=1,2,3$, defined as above, we have

$$
\begin{aligned}
& \left.\frac{d S}{d t}\right|_{S=0}=\Lambda>0,\left.\frac{d E}{d t}\right|_{E=0}=\frac{\beta S I}{1+m I} \geq 0,\left.\frac{d I}{d t}\right|_{I=0}=\epsilon E \geq 0, \\
& \left.\frac{d R}{d t}\right|_{R=0}=\gamma I+u_{1} E+\frac{u_{2} I}{1+\alpha I}+\theta S \geq 0 .
\end{aligned}
$$

Notice that all above rates are non-negative on bounding planes of the non-negative cone of $\mathbb{R}^{4}$. Thus if we begin in the interior of this cone, we shall always remain in this cone as the direction of the vector field is inward on all the bounding planes. Thus all the solutions of model system (1) are positive. Further from (1), note that the total population $N=$ $S+E+I+R$ follows,

$$
\frac{d N}{d t}=\Lambda-\mu N-d I \leq \Lambda-\mu N
$$


Thus $\limsup _{t \rightarrow \infty} N \leq \frac{\Lambda}{\mu}$. Hence all solutions $S, E, I$ and $R$ are bounded by $\frac{\Lambda}{\mu}$. Thus the biologically feasible region of the model system (1) is the following positive invariant set:

$$
\Gamma=\left\{(S, E, I, R) \in \mathbb{R}_{+}^{4} \mid 0 \leq S, E, I, R \leq \frac{\Lambda}{\mu}\right\} .
$$

Since the last equation of model system (1) does not affect the rest equations of the system directly, we consider the following reduced model for further analysis:

$$
\begin{aligned}
& \frac{d S}{d t}=\Lambda-\frac{\beta S I}{1+m I}-\mu S-\theta S, \\
& \frac{d E}{d t}=\frac{\beta S I}{1+m I}-\left(\mu+\epsilon+u_{1}\right) E, \\
& \frac{d I}{d t}=\epsilon E-(\mu+d+\gamma) I-\frac{u_{2} I}{1+\alpha I},
\end{aligned}
$$

with initial conditions $S(0)>0, E(0) \geq 0$ and $I(0) \geq 0$.

\section{Equilibrium Analysis}

In order to explore the dynamics of the model system, the stability of equilibria has been investigated and examined in this section. For this purpose, we first determine the basic reproduction number $R_{0}$ which plays a central role in the disease dynamics and determines the extinction or outbreak of disease. The basic reproduction number is calculated using next-generation matrix method [46] and is given as,

$$
R_{0}=\frac{\epsilon \beta \Lambda}{(\mu+\theta)\left(\mu+\epsilon+u_{1}\right)\left(\mu+d+\gamma+u_{2}\right)} .
$$

Model system (2) has the following equilibria:

(i) disease free equilibrium $E_{1}=\left(\frac{\Lambda}{\mu+\theta}, 0,0\right)$, which exists always and

(ii) infected equilibrium $E_{2}=\left(S_{*}, E_{*}, I_{*}\right)$, which exists whenever $R_{0}>1$,

here $S_{*}=\frac{\Lambda\left(1+m I_{*}\right)}{(\mu+\theta)+(m(\mu+\theta)+\beta) I_{*}}, E_{*}=\frac{\beta S_{*} I_{*}}{\left(1+m I_{*}\right)\left(\mu+\epsilon+u_{1}\right)}$ and $I_{*}=$ $\frac{-B+\sqrt{B^{2}-4 A C}}{2 A}$ is positive root of the following quadratic equation:

$$
f(I)=A I^{2}+B I+C=0 .
$$

Here, $A=\alpha(\mu+d+\gamma)(m(\mu+\theta)+\beta), B=(\mu+d+\gamma)[m(\mu+\theta)+\beta+\alpha(\mu+\theta)]+$ $u_{2}(m(\mu+\theta)+\beta)-\frac{\epsilon \alpha \beta \Lambda}{\left(\mu+\epsilon+u_{1}\right)}$ and $C=(\mu+\theta)\left(\mu+d+\gamma+u_{2}\right)\left(1-R_{0}\right)$. Notice that $A$ is always positive and $C<0$ whenever $R_{0}>1$, thus $f(I)=0$ will have a unique positive root $I_{*}=\frac{-B+\sqrt{B^{2}-4 A C}}{2 A}$. Hence, for $R_{0}>1$ there is a unique infected equilibrium point $E_{2}$.

Remark 1 Note that, when $R_{0}<1$ i.e. $C>0$ along with $B<0$ and $B^{2}-4 A C>0$, then there exist two positive roots of $f(I)=0$ and hence there are two infected equilibrium $E_{3}$ and $E_{4}$ for the model system (2). For $B^{2}-4 A C=0$ both these $E_{3}$ and $E_{4}$ merge into single positive root of $f(I)=0$, giving unique infected equilibrium. 
Remark 2 It is important to mention here that the basic reproduction number $R_{0}$ obtained in this case depends on self-protection parameter $\theta$. Also, $\frac{\partial R_{0}}{\partial \theta}<0$, and hence increase in self protection rate can help in reducing the load of infective in the population. This effect is similar to vaccination effect in epidemiological models.

\section{Local Analysis of Equilibrium Points $E_{1}$ and $E_{2}$}

In the following, we shall establish the local stability of equilibrium points of the model system (2) using the variational matrix given as:

$$
J=\left(\begin{array}{ccc}
-\left(\mu+\theta+\frac{\beta I}{1+m I}\right) & 0 & -\frac{\beta S}{(1+m I)^{2}} \\
\frac{\beta I}{1+m I} & -\left(\mu+\epsilon+u_{1}\right) & \frac{\beta S}{(1+m I)^{2}} \\
0 & \epsilon & -\left(\mu+d+\gamma+\frac{u_{2}}{(1+\alpha I)^{2}}\right)
\end{array}\right) .
$$

Theorem 1 (i) The disease free equilibrium $E_{1}$ of the system (2) is locally asymptotically stable if $R_{0}<1$ and is unstable if $R_{0}>1$.

(ii) If $R_{0}>1$, then the system (2) has unique infected equilibrium point $E_{2}$ and in this case $E_{2}$ is locally asymptotically stable provided $A_{1} B_{1}-C_{1}>0$, where $A_{1}, B_{1}$ and $C_{1}$ are mentioned below.

Proof (i) Follows from Theorem 2 [46].

(ii) The characteristic equation of $J$ at infected equilibrium $E_{2}$ is

$$
\lambda^{3}+A_{1} \lambda^{2}+B_{1} \lambda+C_{1}=0 .
$$

Here $A_{1}=\left(3 \mu+d+\gamma+\theta+\epsilon+u_{1}\right)+\frac{u_{2}}{\left(1+\alpha I_{*}\right)^{2}}+\frac{\beta I_{*}}{\left(1+m I_{*}\right)}, B_{1}=(2 \mu+\theta+\epsilon+$ $\left.u_{1}+\frac{\beta I_{*}}{\left(1+m I_{*}\right)}\right)\left(\mu+d+\gamma+\frac{u_{2}}{\left(1+\alpha I_{*}\right)^{2}}\right)+\left(\mu+\epsilon+u_{1}\right)\left(\mu+\theta+\frac{\beta I_{*}}{1+m I_{*}}\right)-\frac{\epsilon \beta S_{*}}{\left(1+m I_{*}\right)^{2}}$ and $C_{1}=\left(\mu+\epsilon+u_{1}\right)\left(\mu+\theta+\frac{\beta I_{*}}{1+m I_{*}}\right)\left(\mu+d+\gamma+\frac{u_{2}}{\left(1+\alpha I_{*}\right)^{2}}\right)+\frac{\epsilon \beta S_{*}(\mu+\theta)}{\left(1+m I_{*}\right)^{2}}$. Clearly $A_{1}>0$, $C_{1}>0$ and if $A_{1} B_{1}-C_{1}>0$ then Routh-Hurwitz criterion ensures that all the roots of characteristic equation are with negative real parts. Thus, Hartman-Grobman Theorem [35] guarantees the local asymptotic stability of infected equilibrium $E_{2}$ when $R_{0}>1$ provided $A_{1} B_{1}-C_{1}>0$.

\section{Global Analysis of Equilibrium Points $E_{1}$ and $E_{2}$}

To establish the global stability of disease free equilibrium, we follow the method discussed in Castillo-Chavez et al. [11]. Consider, if system (2) can be written of the form as:

$$
\begin{aligned}
& \frac{d X}{d t}=F(X, Y), \\
& \frac{d Y}{d t}=G(X, Y) \text { with } G(X, 0)=0,
\end{aligned}
$$

where $X \in \mathbb{R}^{n_{1}}$ denotes the uninfected population comparments and $Y \in \mathbb{R}^{n_{2}}$ denotes the infected population comparments, $n_{1}$ and $n_{2}$ are positive integers. Let $U_{0}=\left(X_{0}, 0\right)$ is disease free equilibrium of system (3). Consider the following two assumptions:

$\left(H_{1}\right)$ For $\frac{d X}{d t}=F(X, 0), X_{0}$ is globally asymptotically stable. 
$\left(H_{2}\right) G(X, Y)=\mathcal{A} Y-\widehat{G}(X, Y), \widehat{G}(X, Y) \geq 0$ for $(X, Y) \in \Gamma$, where $\mathcal{A}=D_{Y} G\left(X_{0}, 0\right)$ is an M-matrix (stable matrix with non-negative off diagonal elements) and $\Gamma$ is biological feasible region.

Theorem 2 [11] The disease free equilibrium $U_{0}=\left(X_{0}, 0\right)$ of (3) is globally asymptotically stable for $R_{0}<1$ provided the assumptions $\left(H_{1}\right)$ and $\left(H_{2}\right)$ are satisfied.

Now to show the global stability of disease free equilibrium $E_{1}$, we consider the model system (2) in the following form:

$$
\begin{aligned}
& F(X, Y)=\Lambda-\frac{\beta S I}{1+m I}-\mu S-\theta S, \\
& G(X, Y)=\left(\frac{\beta S I}{1+m I}-\left(\mu+\epsilon+u_{1}\right) E, \quad \epsilon E-(\mu+d+\gamma) I-\frac{u_{2} I}{1+\alpha I}\right)^{T},
\end{aligned}
$$

with $G(X, 0)=0$ where $X=S$ and $Y=(E, I)^{T}$. The disease free equilibrium of system (2) is $U_{0}=E_{1}=\left(X_{0}, 0\right)$ with $X_{0}=\frac{\Lambda}{\mu+\theta}$. Clearly $X_{0}$ is globally asymptotically stable for $\frac{d X}{d t}=F(X, 0)$ as $X \rightarrow \frac{\Lambda}{\mu+\theta}$ when $t \rightarrow \infty$. Further

$G(X, Y)=\left(\begin{array}{cc}-\left(\mu+\epsilon+u_{1}\right) & \frac{\beta \Lambda}{(\mu+\theta)} \\ \epsilon & -\left(\mu+d+\gamma+u_{2}\right)\end{array}\right)\left(\begin{array}{c}E \\ I\end{array}\right)-\left(\begin{array}{c}\beta I\left(\frac{\Lambda(1+m I)-(\mu+\theta) S}{(\mu+\theta)(1+m I)}\right) \\ \frac{u_{2} I}{(1+\alpha I)}-u_{2} I\end{array}\right)$.

Consider $\mathcal{A}=\left(\begin{array}{cc}-\left(\mu+\epsilon+u_{1}\right) & \frac{\beta \Lambda}{(\mu+\theta)} \\ \epsilon & -\left(\mu+d+\gamma+u_{2}\right)\end{array}\right)$ and $\widehat{G}(X, Y)=\left(\begin{array}{c}K \\ \frac{-u_{2} \alpha I^{2}}{(1+\alpha I)}\end{array}\right)$.

Here $\widehat{G_{1}}(X, Y)=K=\frac{\beta I}{(\mu+\theta)(1+m I)}\left\{m \Lambda I+(\mu+\theta)\left(\frac{\Lambda}{(\mu+\theta)}-S\right)\right\} \geq 0$ as $0<S \leq$ $\frac{\Lambda}{(\mu+\theta)}$ and $\widehat{G_{2}}(X, Y)=\frac{-u_{2} \alpha I^{2}}{(1+\alpha I)} \leq 0$. Notice that assumption $\left(H_{1}\right)$ is satisfied but $\left(H_{2}\right)$ will be satisfied provided $\alpha=0$.

We summarise the above discussion in the following.

Theorem 3 The disease free equilibrium $E_{1}$ of system (2) is globally asymptotically stable in $\Gamma$ when $R_{0}<1$ and $\alpha=0$.

Remark 3 If $\alpha \neq 0$, the global stability of disease free equilibrium $E_{1}$ of system (2) may not be guaranteed when $R_{0}<1$. The treatment rate $u_{2}$ is taken to be non-vanishing. So, the saturation in treatment may lead to endemicity even if the $R_{0}<1$.

Remark 4 Note that when $R_{0}<1$ and $\alpha=0(A=0, B>0$ and $C>0)$ then there exists only disease free equilibrium and that will be globally asymptotically stable.

The global stability of the infected equilibrium $E_{2}$ is established by constructing a Lyapunov function and applying Lyapunov LaSalle Theorem [34]. The details are provided in Appendix A.

\section{Bifurcation Analysis at $R_{0}=1$}

In this section, we shall explore the local analysis of disease free equilibrium at $R_{0}=1$ and determine the direction of bifurcation. For this purpose, center manifold theory is applied as discussed in Castillo-Chavez and Song [12] and as reproduced in Appendix B. 
Consider $\beta$ as a bifurcation parameter at $R_{0}=1$. So at $\beta=\beta^{*}, R_{0}=1$ gives $\beta^{*}=$ $\frac{(\mu+\theta)\left(\mu+\epsilon+u_{1}\right)\left(\mu+\delta+\gamma+u_{2}\right)}{\epsilon \Lambda}$. The model system (2) can be written as:

$$
\begin{aligned}
& \frac{d S}{d t}=\Lambda-\frac{\beta S I}{1+\alpha_{1} I}-\mu S-\theta S:=f_{1}, \\
& \frac{d E}{d t}=\frac{\beta S I}{1+\alpha_{1} I}-\left(\mu+\epsilon+u_{1}\right) E:=f_{2}, \\
& \frac{d I}{d t}=\epsilon E-(\mu+\delta+\gamma) I-\frac{u_{2} I}{1+\alpha_{2} I}:=f_{3},
\end{aligned}
$$

with the disease free equilibrium $E_{1}$. The linearization matrix corresponding to model system (4) at $\left(E_{1}, \beta^{*}\right)$ is given as:

$$
J_{E_{1}}\left(\beta^{*}\right)=\left(\begin{array}{ccc}
-(\mu+\theta) & 0 & -\frac{\beta^{*} \Lambda}{(\mu+\theta)} \\
0 & -\left(\mu+\epsilon+u_{1}\right) & \frac{\beta^{*} \Lambda}{(\mu+\theta)} \\
0 & \epsilon & -\left(\mu+d+\gamma+u_{2}\right)
\end{array}\right) .
$$

Matrix $J_{E_{1}}\left(\beta^{*}\right)$ has an eigenvalue zero at $R_{0}=1$ which is simple eigenvalue and other two eigenvalues are negative. The right and left eigenvectors corresponding to the zero eigenvalue are $w=\left[-\frac{\beta^{*} \Lambda}{(\mu+\theta)}, \frac{\beta^{*} \Lambda}{\left(\mu+\epsilon+u_{1}\right)},(\mu+\theta)\right]^{T}$ and $v=\left[0,1, \frac{\beta^{*} \Lambda}{(\mu+\theta)\left(\mu+d+\gamma+u_{2}\right)}\right]$ respectively. The non zero second order partial derivatives of $f_{1}, f_{2}$ and $f_{3}$ at $\left(E_{1}, \beta^{*}\right)$ are given as:

$$
\begin{aligned}
& \frac{\partial^{2} f_{2}}{\partial I \partial S}=\beta^{*}, \quad \frac{\partial^{2} f_{2}}{\partial S \partial I}=\beta^{*}, \quad \frac{\partial^{2} f_{2}}{\partial I^{2}}=-2 \alpha_{1} \beta^{*} \frac{\Lambda}{(\mu+\theta)}, \\
& \frac{\partial^{2} f_{3}}{\partial I^{2}}=-2 \alpha_{2} u_{2}, \quad \text { and } \quad \frac{\partial^{2} f_{2}}{\partial I \partial \beta}=\beta^{*} \frac{\Lambda}{(\mu+\theta)} .
\end{aligned}
$$

Hence, we get $a=2 \beta^{*} \Lambda\left[\frac{\alpha u_{2}(\mu+\theta)}{\left(\mu+d+\gamma+u_{2}\right)}-\left(\beta^{*}+m(\mu+\theta)\right)\right]$ and $b=\Lambda$. Clearly $a>0$ if $\alpha>\alpha_{*}:=\frac{\left(\beta^{*}+m(\mu+\theta)\right)\left(\mu+d+\gamma+u_{2}\right)}{u_{2}(\mu+\theta)}$. Using Theorem 8 given in Appendix B, we get the following.

Theorem 4 (i) If $\alpha>\alpha_{*}$, then backward bifurcation occurs at $R_{0}=1$ for the model system (2).

(ii) If $\alpha<\alpha_{*}$, as $R_{0}$ crosses unity then disease free equilibrium changes its stability form stable to unstable and there exists a locally asymptotically infected equilibrium when $R_{0}>1$ i.e. direction of bifurcation is forward (transcritical) at $R_{0}=1$.

Remark 5 We note from above that when $R_{0}>1$, a unique infected equilibrium for the model system (2) exits which is locally asymptotically stable. Thus the results of the Theorem 1 follows, and hence the condition of Theorem 1(ii) is also fulfilled.

Remark 6 From Theorem 4, we observe that saturation in medical treatment leads to backward bifurcation at $R_{0}=1$. This happens because infected individuals increase during the epidemic outbreak and all infective do not get treatment simultaneously beyond medical capacity. This delay in treatment process forces infective to stay for a longer time in the population even if $R_{0}<1$. Thus, limitation in medical facilities causes backward bifurcation i.e. the existence of more infected equilibria for $R_{0}<1$. In the following, we numerically validate the obtained results for a representative set of parameters. 


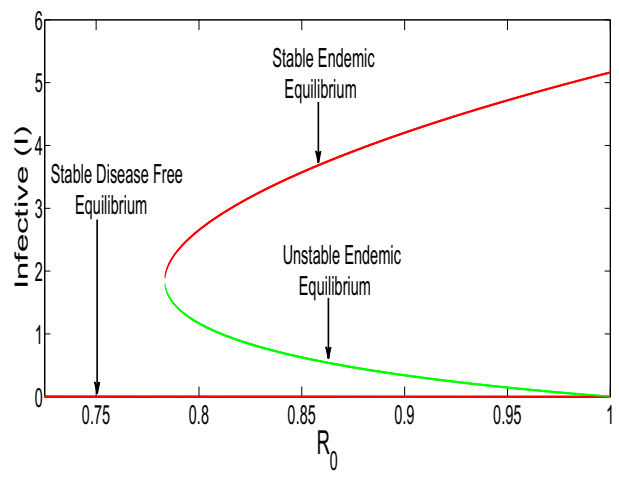

(a)

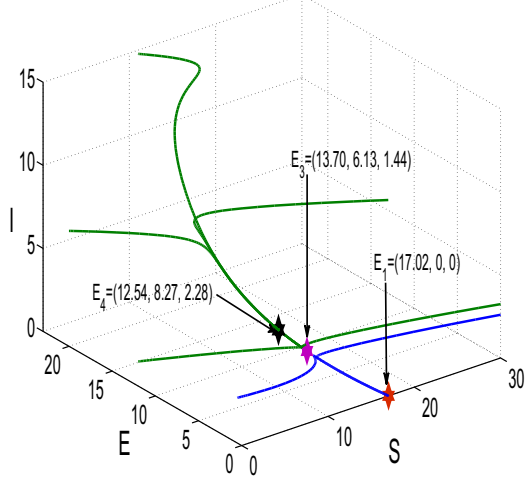

(b)

Fig. 2 a Occurrence of backward bifurcation at $R_{0}=1$ when $\alpha>\alpha_{*}$. b Existence of three equilibria $E_{1}, E_{3}$ and $E_{4}$ when $R_{0}<1$, with solution trajectories starting from different initial points approaching to $E_{1}$ and $E_{4}$ showing the local stability and instability of $E_{3}$

\section{Numerical Results for the Model System (2)}

Example 1 Consider a representative set of parameters as: $\Lambda=4, \beta=0.045, m=0.1, \mu=$ $0.01, u_{1}=0.1, \epsilon=0.017, d=0.01, \gamma=0.01, u_{2}=0.1$ and $\alpha=0.95$. We vary $\theta \in$ $[0.175342,0.25]$ so that $0.7129 \leq R_{0} \leq 1$. The Fig. 2a shows the existence of backward bifurcation in this case.

Further, in Fig. 2b we show the solution trajectories approaching to different equilibria starting with different initial values. We fix $\theta=0.225$ and other parameters same as above. In this case $R_{0}=0.788<1$ and $\alpha_{*}=0.4456<\alpha=0.95$, and hence there exist a disease free equilibrium $E_{1}=(17.02,0,0)$ and two infected equilibria $E_{3}=(13.70,6.13,1.44)$ and $E_{4}=(12.54,8.27,2.28)$. We graphically represent the local asymptotic stability of disease free equilibrium $E_{1}$ and infected equilibrium $E_{4}$ along with instability of infected equilibrium $E_{3}$ in Fig. 2b. Thus, Theorem 4(i) is verified.

Example 2 In this example, we graphically show the occurrence of transcritical bifurcation at $R_{0}=1$ when $\alpha<\alpha_{*}$ as given in Theorem 4(ii). For this we consider parameter $\alpha=0.4$, $\theta \in[0.135,0.25]$ (i.e. $0.7129 \leq R_{0} \leq 1.2782$ ) and rest of the parameters as taken in Example 1. One can easily see that $\alpha=0.4<\alpha_{*}=0.4456$ and the corresponding plot for transcritical bifurcation at $R_{0}=1$ is given in Fig. 3a. Further, for a fixrd $\theta=0.14$ and other parameters as above, we note that $R_{0}=1.235>1$, and the condition $A_{1} B_{1}-C_{1}=0.0176>0$ of Theorem 4(ii) holds showing that the unique infected equilibrium exists and is also locally stable for $R_{0}>1$.

Example 3 Again choose $\Lambda=1, \beta=0.005, m=0.9, \mu=0.001, u_{1}=0.01, \epsilon=$ $0.01, d=0.01, \gamma=0.01, u_{2}=0.1, \theta=0.0013$ and $\alpha=0.95$ so that $R_{0}=8.55>1$. A unique infected equilibrium $E_{2}=(135.13,32.70,11.00)$ is obtained in this case. The corresponding global asymptotic stability of $E_{2}$ is shown in Fig. $3 \mathrm{~b}$ for different initial conditions.

Further, in this case, to see the impact of self-protection on the disease dynamics, we plotted the solution trajectories for different values of $\theta$. The corresponding results are given in Fig. 4. We infer from Fig. 4 that the higher self-protection significantly reduces the count 


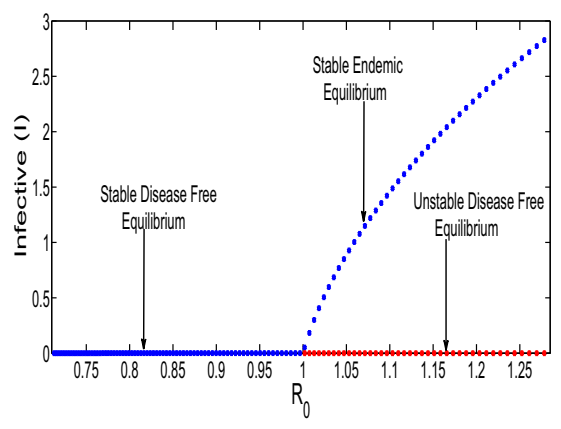

(a)

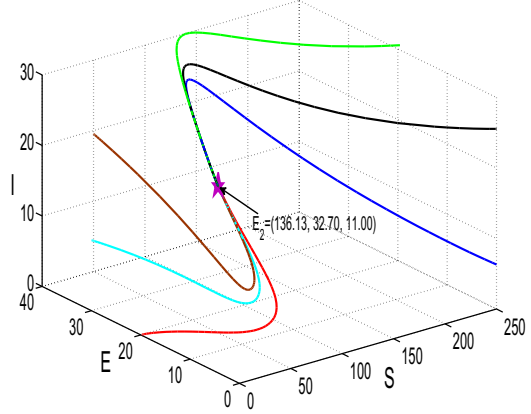

(b)

Fig. 3 a Occurrence of transcritical bifurcation at $R_{0}=1$ when $\alpha<\alpha_{*}$. b Global asymptotic stability of unique infected equilibrium $E_{2}$ when $R_{0}>1$
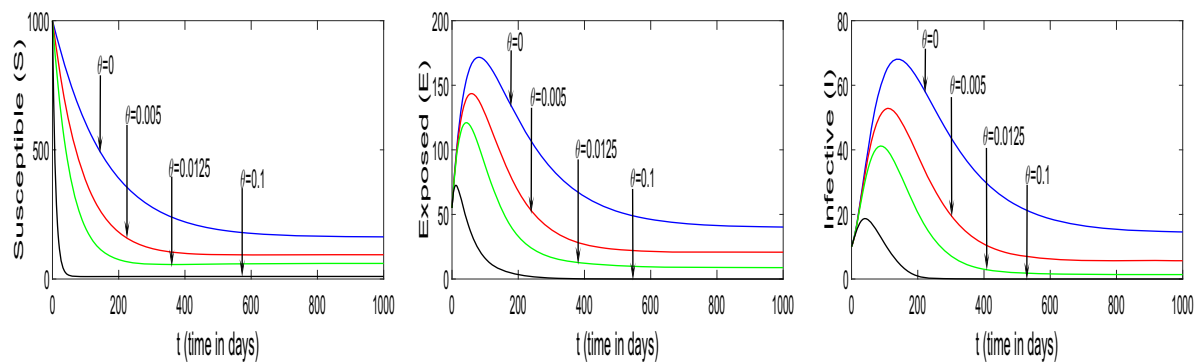

Fig. 4 Effect of the self-protection $(\theta)$ on the population

of infective population as well as exposed (high risk group) population. It can be also noted that the higher self-protection not only reduces the count of infective but also minimizes the duration of the disease prevalence (compare the graphs of $I$ for $\theta=0$ and $\theta=0.1$ ). Hence, the self-protection plays an important role in reducing the cumulative disease burden.

Remark 7 From the basic reproduction number $R_{0}=\frac{\epsilon \beta \Lambda}{(\mu+\theta)\left(\mu+\epsilon+u_{1}\right)\left(\mu+d+\gamma+u_{2}\right)}$, we note that $\frac{\partial R_{0}}{\partial \theta}<0$ and $\frac{\partial R_{0}}{\partial u_{1}}<0$ i.e. $R_{0}$ decreases when self-protection $\theta$ and/or screening effort $u_{1}$ increases. It is clear from Fig. 5a that whenever $\theta$ and/or $u_{1}$ approaches to zero, surface of $R_{0}$ increases and crosses unit surface $\left(R_{0}=1\right)$ that measures the outbreak of disease. This implies that higher level of self-protection $\theta$ and screening effort $u_{1}$ may reduce the prevalence of disease by bringing $R_{0}<1$. We also note $\frac{\partial R_{0}}{\partial u_{2}}<0$, and hence as shown in Fig. 5b, similar conclusion can be drawn that higher level of self-protection $\theta$ and treatment effort $u_{2}$ may reduce the prevalence of disease. Further, to see the effect of both controls $\left(u_{1}\right.$ and $u_{2}$ ) on $R_{0}$, we plot the corresponding result in Fig. 5c. We observe that when screening and treatment simultaneously increase then surface of $R_{0}$ crosses the $R_{0}=1$ surface and goes down to below one that gives the extinction of disease. Thus we, analytically and numerically, observe that self-protection, screening and treatment suppress the disease burden during the course of epidemic. 


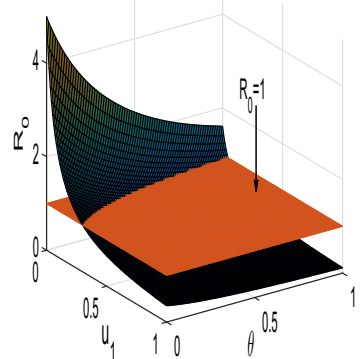

(a)

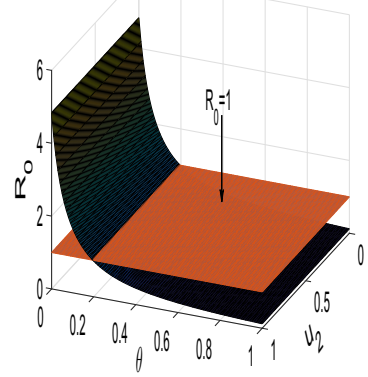

(b)

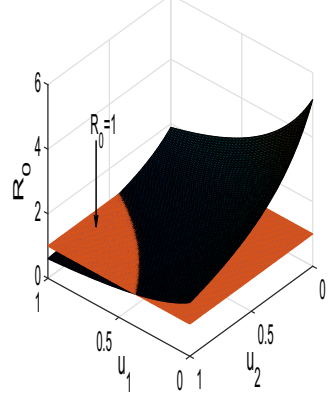

(c)

Fig. 5 a $R_{0}$ with respect to screening effort $u_{1}$ and self-protection $\theta$. b $R_{0}$ with respect to treatment $u_{2}$ and self-protection $\theta$. c $R_{0}$ with respect to screening effort $u_{1}$ and treatment $u_{2}$

\section{Optimal Control Problem}

In this section, we formulate an optimal control problem corresponding to the model (1) by considering screening and treatment as control interventions. Implementation of such control interventions requires huge manpower, medical infrastructure and involves related costs. In the following, we briefly discuss these control interventions.

(i) Screening effort on exposed population: As found in practice the screening or identification of symptomatic individuals i.e. the infective population is comparatively easier than the exposed individuals (asymptomatic) due to their clinical symptoms related to infection. Thus it is really challenging to detect and treat these asymptomatic individuals. We, in this control problem, implement screening effort on exposed individuals. Screening efforts are expensive and difficult to employ and expense increases steeply for covering a larger proportion of population [36]. As resources and funds are limited, the efforts should be optimally used. Thus in model system (1), $u_{1}$, the screening rate is considered as control variable $u_{1}(t)$. The aim is to investigate an optimal way of screening that minimizes new infection as well as cost. Bounds on controls come naturally due to resource limitation as $0 \leq u_{1}(t) \leq u_{1 \text { max }}$.

(ii) Recovery of infective via treatment: Treatment helps in reducing the consequences of disease and also increase productive capacity. In model system (1), we incorporate a saturated treatment as $\frac{u_{2} I}{1+\alpha I}$ where $u_{2}$ is constant treatment rate. As there is limitation on medical facilities and related efforts and also the implementation of treatment involves a significant cost, we consider constant treatment rate $u_{2}$ as control variable $u_{2}(t)$ for model system (1) with $0 \leq u_{2}(t) \leq u_{2 \max }$. Thus the aim is to find an optimal path for treatment policy that minimizes infective and cost due to disease and treatment.

The admissible set of control functions $u_{1}(t)$ and $u_{2}(t)$ is defined as:

$$
U=\left\{\left(u_{1}(t), u_{2}(t)\right) \mid\left(u_{1}(t), u_{2}(t)\right) \in\left[0, u_{1 \max }\right] \times\left[0, u_{2 \max }\right], t \in[0, T]\right\} .
$$

Here, $u_{1}(t)$ and $u_{2}(t)$ are measurable and bounded functions and $T$ is the final time of the applied control interventions. Our main objective of this study is to investigate such optimal control functions $u_{1}^{*}(t)$ and $u_{2}^{*}(t)$ that not only minimize the total cost but also cumulative count of infection. 
Now consider the following cost functional that has to be minimized

$$
J_{1}\left[u_{1}(t), u_{2}(t)\right]=\int_{0}^{T}\left[A_{1} E(t)+A_{2} I(t)+B_{1} u_{1}^{4}(t)+B_{2} u_{2}^{2}(t)\right] d t
$$

with subject to the model system

$$
\begin{aligned}
\frac{d S}{d t} & =\Lambda-\frac{\beta S I}{1+m I}-\mu S-\theta S, \\
\frac{d E}{d t} & =\frac{\beta S I}{1+m I}-\left(\mu+\epsilon+u_{1}(t)\right) E, \\
\frac{d I}{d t} & =\epsilon E-(\mu+d+\gamma) I-\frac{u_{2}(t) I}{1+\alpha I}, \\
\frac{d R}{d t} & =\gamma I-\mu R+u_{1}(t) E+\theta S+\frac{u_{2}(t) I}{1+\alpha I},
\end{aligned}
$$

along with initial conditions $S(0)>0, E(0) \geq 0, I(0) \geq 0$ and $R(0) \geq 0 . J_{1}$ represents the total cost incurred due to disease burden and applied intervention policies. The integrand $L\left(S, E, I, R, u_{1}, u_{2}\right)=A_{1} E(t)+A_{2} I(t)+B_{1} u_{1}^{4}(t)+B_{2} u_{2}^{2}(t)$ represents the weighted current cost at time $t$. Positive weight constants $A_{1}, A_{2}, B_{1}$ and $B_{2}$ represent the dual nature that balance units of integrand and related cost. In the following, we describe the various components given in $L$.

- Terms $A_{1} E(t)$ and $A_{2} I(t)$ represent the cost incurred due to disease that includes the loss of man-hour, opportunity loss (productivity loss due to sickness), loss generated in searching treatment and protection, patient caring, and related wealth etc. $[19,26,28,32$, $33,49]$.

- The third term $B_{1} u_{1}^{4}(t)$ of $L$ describes the cost involved in screening effort during the outbreak of an epidemic. As stated above, screening and related efforts are very difficult and it is an expensive task to cover a large proportion of the population. Also, cost of screening not only increases but also goes more steeply to cover the same fraction of population with high-density i.e. screening for a higher proportion of the population will be costlier than the lower one. To account it in cost, the nonlinearity of order four of $u_{1}^{4}(t)$ is considered for screening cost and related efforts [32,33,36,50].

- The term $B_{2} u_{2}^{2}(t)$ of $L$ denotes the cost incurred in treatment strategy and related efforts such as medicine, diagnosis charges and hospitalization etc. in the period of providing treatment to infective. On the basis of severity and effect of treatment, we consider second order nonlinearity in the incurred cost of treatment $[19,27,28,32,33]$.

Further for the sake of convenience, we will consider $u_{1}(t)=u_{1}$ and $u_{2}(t)=u_{2}$.

\section{Existence of Pair of Optimal Controls}

Here, we shall show the existence of optimal control functions which minimize the cost functional in a finite time period. For this purpose, we follow the method discussed in [17, 19,20]. The following result ensures the existence of optimal control functions.

Theorem 5 There exists a pair of optimal controls $u_{1}^{*}$ and $u_{2}^{*}$ in $U$ such that $J_{1}\left[u_{1}^{*}, u_{2}^{*}\right]=$ $\min \left[J_{1}\left[u_{1}, u_{2}\right]\right]$ corresponding to the control system (5)-(6).

Proof To show, we follow Theorem 4.1 pp. 68 in [17] where following conditions must be satisfied: 
(i) The set of solutions to system (6) with control variables in $U$ is non empty.

(ii) $U$ is closed and convex and state system can be written as linear function of control variables with coefficients depending on time and state variables.

(iii) Integrand $L$ of equation (6) is convex on $U$ and $L\left(S, E, I, R, u_{1}, u_{2}\right) \geq g\left(u_{1}, u_{2}\right)$ where $g$ is continuous and $\left|\left(u_{1}, u_{2}\right)\right|^{-1} g\left(u_{1}, u_{2}\right) \rightarrow \infty$ as $\left|\left(u_{1}, u_{2}\right)\right| \rightarrow \infty$, here | . | represents the $l^{2}(0, T)$ norm.

As seen in section "Positivity and Boundedness of Solutions", we note that solutions of system (6) are bounded for each bounded control variables in $U$. Clearly the right hand side functions of system (6) satisfies the Lipschitz condition with respect to state variables. Hence condition $(i)$ is achieved following Picard-Lindelöf theorem [13].

By definition, the control set $U$ is closed and convex, and model system (6) is linear in control variables $u_{1}$ and $u_{2}$ with coefficients depending on state variables. Thus condition (ii) is satisfied. Integrand $L$ is convex due to the biquadratic and quadratic nature of control variables $u_{1}$ and $u_{2}$. Further, $L\left(S, E, I, R, u_{1}, u_{2}\right)=A_{1} E+A_{2} I+B_{1} u_{1}^{4}+B_{2} u_{2}^{2} \geq B_{1} u_{1}^{4}+$ $B_{2} u_{2}^{2}$. Now choosing $c_{1}=\min \left(B_{1}, B_{2}\right)>0$ and $g\left(u_{1}, u_{2}\right)=c_{1}\left(u_{1}^{4}+u_{2}^{2}\right)$ then we get $L\left(S, E, I, R, u_{1}, u_{2}\right) \geq g\left(u_{1}, u_{2}\right)$, and notice that $g$ is continuous and satisfy $\left|\left(u_{1}, u_{2}\right)\right|^{-1}$ $g\left(u_{1}, u_{2}\right) \rightarrow \infty$ whenever $\left|\left(u_{1}, u_{2}\right)\right| \rightarrow \infty$ thus condition (iii) is fulfilled. Hence, there is a control pair $u_{1}^{*}$ and $u_{2}^{*}$ such that $J_{1}\left[u_{1}^{*}, u_{2}^{*}\right]=\min \left[J_{1}\left[u_{1}, u_{2}\right]\right]$.

\section{Characterization of Optimal Control Functions}

In this part, we shall derive the necessary conditions for optimal control functions using Pontryagin's Maximum Principle and also characterize the optimal controls for system (5)-(6) $[17,21]$. As Pontryagin's Maximum Principle plays an important role in adjoining the cost functional with state equations by incorporating adjoint variables. First, we define Hamiltonian as,

$$
H\left(S, E, I, R, u_{1}, u_{2}, \lambda\right)=L\left(S, E, I, R, u_{1}, u_{2}\right)+\lambda_{1} \frac{d S}{d t}+\lambda_{2} \frac{d E}{d t}+\lambda_{3} \frac{d I}{d t}+\lambda_{4} \frac{d R}{d t} .
$$

Here $\lambda=\left(\lambda_{1}, \lambda_{2}, \lambda_{3}, \lambda_{4}\right)$ is known as adjoint variable.

Theorem 6 Let $u_{1}^{*}$ and $u_{2}^{*}$ be optimal control functions and $S^{*}, E^{*}, I^{*}$ and $R^{*}$ be corresponding state variables of the control problem (5)-(6). Then there exist adjoint variable $\lambda=\left(\lambda_{1}, \lambda_{2}, \lambda_{3}, \lambda_{4}\right) \in \mathbb{R}^{4}$ which satisfy the following canonical equations:

$$
\begin{aligned}
\frac{d \lambda_{1}}{d t}= & \left(\mu+\theta+\frac{\beta I}{1+m I}\right) \lambda_{1}-\frac{\beta I}{1+m I} \lambda_{2}-\theta \lambda_{4}, \\
\frac{d \lambda_{2}}{d t}= & -A_{1}+\left(\mu+\epsilon+u_{1}\right) \lambda_{2}-\epsilon \lambda_{3}-u_{1} \lambda_{4}, \\
\frac{d \lambda_{3}}{d t}= & -A_{2}+\frac{\beta S}{(1+m I)^{2}} \lambda_{1}-\frac{\beta S}{(1+m I)^{2}} \lambda_{2}+\left(\mu+d+\gamma+\frac{u_{2}}{(1+\alpha I)^{2}}\right) \lambda_{3} \\
& -\left(\gamma+\frac{u_{2}}{(1+\alpha I)^{2}}\right) \lambda_{4}, \\
\frac{d \lambda_{4}}{d t}= & \mu \lambda_{4},
\end{aligned}
$$

with transversality conditions $\lambda_{1}(T)=0, \lambda_{2}(T)=0, \lambda_{3}(T)=0$ and $\lambda_{4}(T)=0$. 
The corresponding optimal controls $u_{1}^{*}$ and $u_{2}^{*}$ are given as,

$$
u_{1}^{*}=\min \left\{\max \left\{0,\left(\frac{E^{*}}{4 B_{1}}\left(\lambda_{2}-\lambda_{4}\right)\right)^{\frac{1}{3}}\right\}, u_{1 \max }\right\} .
$$

and

$$
u_{2}^{*}=\min \left\{\max \left\{0, \frac{I^{*}}{2 B_{2}\left(1+\alpha I^{*}\right)}\left(\lambda_{3}-\lambda_{4}\right)\right\}, u_{2 \max }\right\} .
$$

Proof Let $u_{1}^{*}$ and $u_{2}^{*}$ be the given optimal controls and $S^{*}, E^{*}, I^{*}$ and $R^{*}$ be corresponding optimal state variables of system (6) that minimize the cost functional (5). Then by Pontryagin's Maximum Principle, there exist adjoint variables $\lambda_{1}, \lambda_{2}, \lambda_{3}$ and $\lambda_{4}$ which satisfy following canonical equations

$$
\frac{d \lambda_{1}}{d t}=-\frac{\partial H}{\partial S}, \frac{d \lambda_{2}}{d t}=-\frac{\partial H}{\partial E}, \frac{d \lambda_{3}}{d t}=-\frac{\partial H}{\partial I} \text { and } \frac{d \lambda_{4}}{d t}=-\frac{\partial H}{\partial R}
$$

with transversality conditions $\lambda_{1}(T)=0, \lambda_{2}(T)=0, \lambda_{3}(T)=0$ and $\lambda_{4}(T)=0$. Here Hamiltonian $H$ is as given in (7). Then (8) is satisfied.

Now from the optimality condition, we have

$$
\frac{\partial H}{\partial u_{i}}=0, \quad \text { at } u_{1}=u_{i}^{*} \text { for } i=1,2 .
$$

Thus, we get

$$
u_{1}^{*}=\left(\frac{E^{*}}{4 B_{1}}\left(\lambda_{2}-\lambda_{4}\right)\right)^{\frac{1}{3}} \text { and } u_{2}^{*}=\frac{I^{*}}{2 B_{2}\left(1+\alpha I^{*}\right)}\left(\lambda_{3}-\lambda_{4}\right) .
$$

Using characteristics of the control space $U$ and above discussion, the optimal controls $u_{1}^{*}$ and $u_{2}^{*}$ are given as in (9) and (10).

\section{Numerical Experimentation with Discussion for the Optimal Control Problem}

In this section, we perform some numerical simulations using MATLAB to validate the analytical results obtained in the previous section.

To investigate the effect of control interventions on the dynamics of disease, we shall implement various combinations of controls such as: implementation of only screening, implementation of only treatment and execution of both. For our numerical simulations, we consider the implementation time period for controls $T=120$ days. Parametric values are considered as: $\Lambda=0.2$ person day ${ }^{-1}$ (Assumed, [19]), $\mu=0.00004$ day $^{-1}$ [19,39], $\beta=0.05$ person $^{-1} \mathrm{day}^{-1}[19], \epsilon=0.1 \mathrm{day}^{-1}[19,23], d=0.05 \mathrm{day}^{-1}[19], \gamma=0.04 \mathrm{day}^{-1}$ [23], $\theta=0.0005$ day $^{-1}$ [28], $m=0.95$ person $^{-1}$, and $\alpha=0.01$ person $^{-1}$. First we solve system (6) numerically using 4th order Runge-Kutta method in MATLAB with initial population size $S(0)=4900, E(0)=80, I(0)=19, R(0)=1$ in absence of screening effort i.e. $u_{1}=0$ as well as absence of treatment i.e. $u_{2}=0$. The corresponding solution trajectories are plotted in Fig. 6a. In the absence of controls, we observe that both exposed and infected individuals initially increase very fast after emerging of the infection. They reach maximum peak quickly and high prevalence of the disease is observed for the almost entire period. This high prevalence not only affects the social well being but also increases transmission probability and causes productivity loss. 


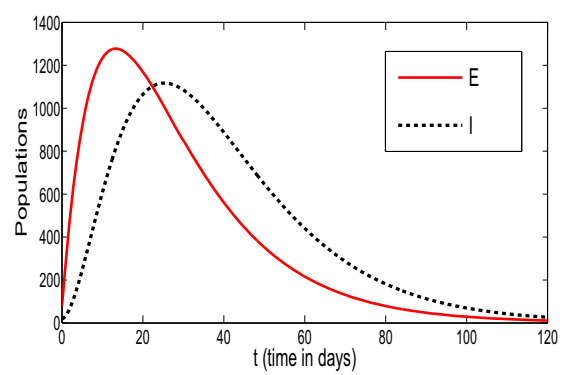

(a)

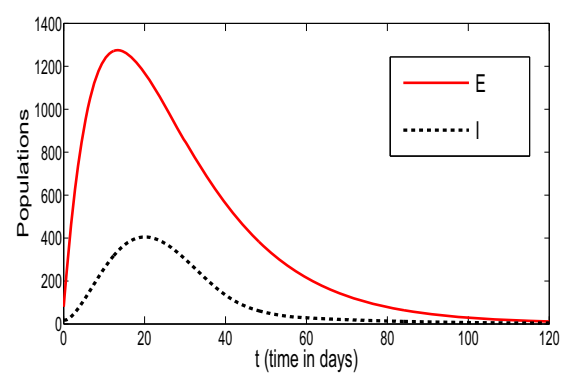

(b)

Fig. 6 a Populations with $u_{1}=0$ and $u_{2}=0$. b Populations with $u_{2}^{*}$ when $u_{1}=0$

To simulate optimality system (12)-(13) numerically, we use the forward-backward sweep method starting with an initial guess for optimal controls and solve the optimal state system forward in time and after that solve the adjoint state system backward in time, due to transversality conditions, using the optimal state variables and initial guess of optimal controls. Then the optimal control functions are updated with the help of adjoint variables and optimal state variables and the same process is continued till a pre-defined convergence criterion is met (for complete details see [38]). The positive weight constants are chosen as $A_{1}=1, A_{2}=5, B_{1}=1000$ and $B_{2}=100$ and here it is assumed that the weight of screening effort is larger than the weight of treatment as screening effort consumes more money than the treatment. Further, we solve the optimality system (12)-(13) for $u_{1}=0$ i.e. screening intervention is not applied and the only treatment is available. Corresponding population distribution are plotted in Fig. $6 \mathrm{~b}$. We observe that treatment effectively reduces the count of infective but growth of exposed individuals is unaffected. Significant decay in the peak of infective along with less prevalence period is obtained in comparison with the case where no controls. The optimal profile of treatment is plotted in Fig. 7(a). Note that for a half time period of epidemic treatment has to be applied with its maximum level of capacity with a gradual reduction for the later period.

Next we simulate the optimality system (12)-(13) when only screening is employed i.e. $u_{2}=0$. For comparison of the effect of these single control interventions, the distribution of exposed and infected individuals is presented in Fig. 8 for these two cases. It is clear from Fig. $8 \mathrm{a}$, b that the only screening policy works better than the only treatment policy during

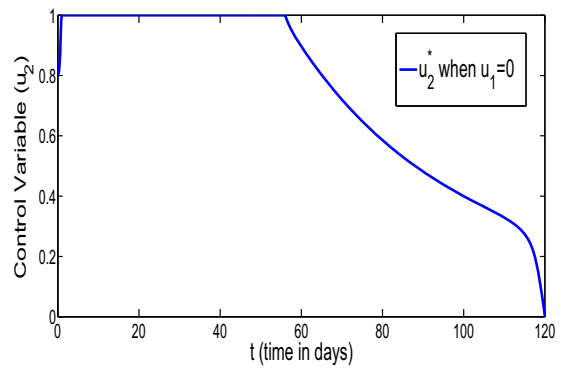

(a)

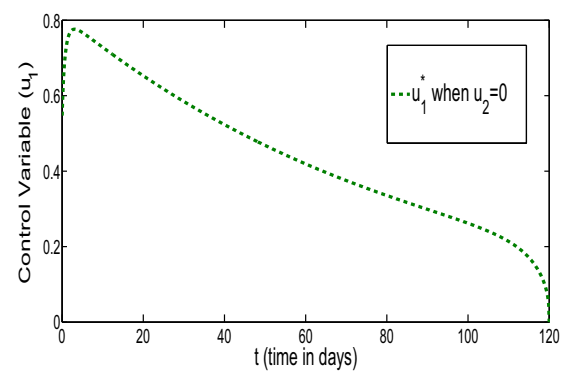

(b)

Fig. 7 a Path of optimal treatment $u_{2}^{*}$ when $u_{1}=0$. b Path of optimal screening $u_{1}^{*}$ when $u_{2}=0$ 


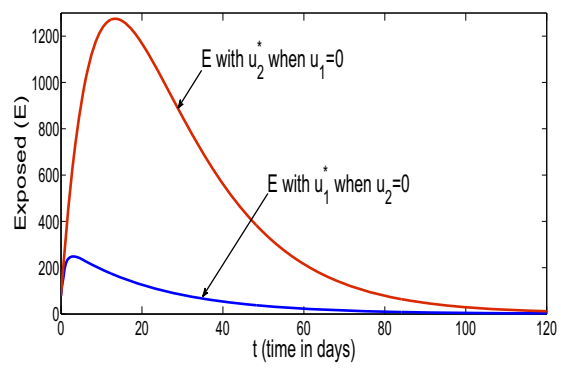

(a)

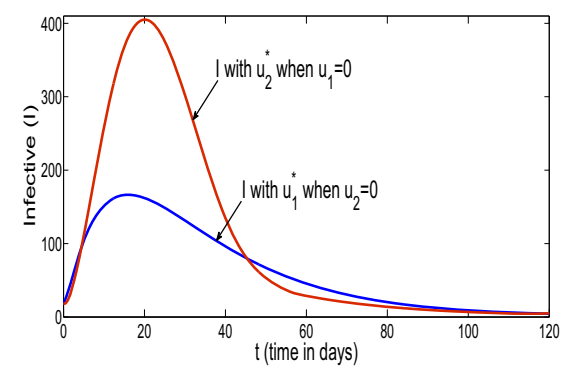

(b)

Fig. 8 a $E$ under optimal controls $u_{1}^{*}$ and $u_{2}^{*}$. b $I$ under optimal controls $u_{1}^{*}$ and $u_{2}^{*}$

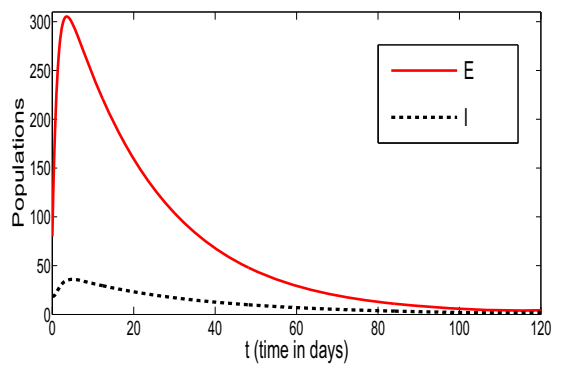

(a)

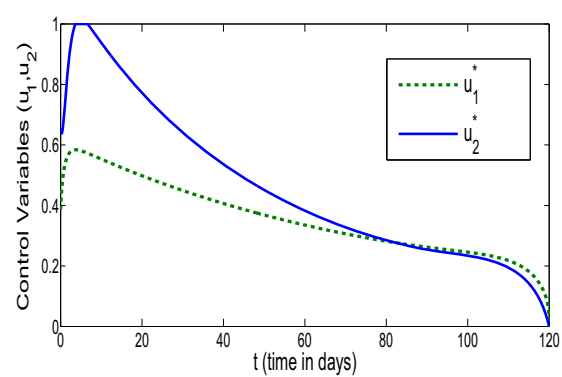

(b)

Fig. 9 a Populations with $u_{1}^{*}$ and $u_{2}^{*}$. b Optimal controls $u_{1}^{*}$ and $u_{2}^{*}$

the course of infection. Impact of screening not only suppresses the count of a cumulative number of infective but also reduces the duration of prevalence. The corresponding optimal profile of screening is plotted in Fig. 7b. Execution of screening with significantly higher potential is required to suppress the cumulative count of infection. From Fig. 8 it is also clear that optimal screening lowers the count of exposed individuals that directly affects the dynamics of infective. Thus numerical findings suggest that future disease burden can be reduced via screening of the infective at the early stage. Thus only screening policy is found better than the only treatment policy.

Further both controls: screening and treatment are considered. Population profiles plotted in Fig. 9a, describe that the combined effect of screening and treatment not only reduces the prevalence of both exposed and infected individuals but also minimizes the duration of disease prevalence. A very small heap on the exposed individuals is observed whereas the count of the infective population is significantly low. Thus among the all three control strategies i.e. only screening, only treatment and combination of screening and treatment, the combined use of screening and treatment works better and reduces the disease transmission and prevalence. The profiles of optimal screening and treatment are plotted in Fig. 9b. We can easily see that first 50 days treatment has to be executed with higher intensity than the screening whereas afterward screening and treatment together work with more or less same impact.

As cost-effectiveness of the applied control interventions is a crucial factor that determines their usefulness, we, thus, performed a comparative study of costs numerically for the three strategies discussed above along with no control case. In Fig. 10, the paths of optimal costs 


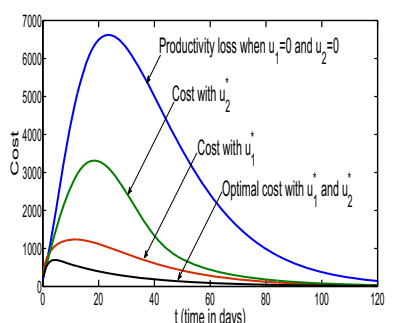

(a)

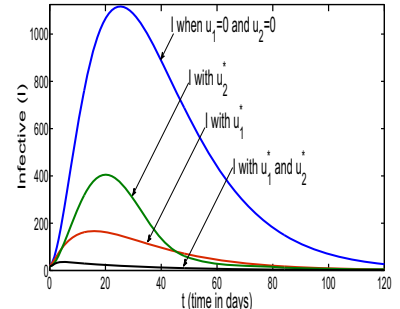

(b)

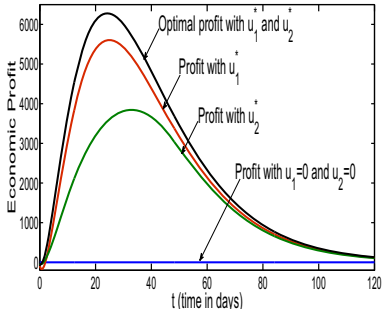

(c)

Fig. 10 a Cost distribution under various control interventions. b $I$ under various control interventions. c Profiles of economic profit under various control interventions

for these four cases, disease burden for the study period along with the 'economic profit' for selecting a certain strategy is plotted. From Fig. 10a note that in the absence of controls, productivity loss is the only contributor to costs and is found to be maximum.

Also, note that comprehensive effect of screening and treatment is less expensive as well as most effective. In between single control policies, screening is found better than the treatment during the course of the epidemic. If screening is not available, treatment works well but at high expense. The infective population remains low when screening is applied and is least when both the screening and treatment are applied. The "economic profit" is calculated by considering the cost of productivity loss due to the presence of infective as total baseline expenditure. Now the costs of different control strategies are calculated. The difference between these two costs is in a sense profit to the government and that profit is plotted in Fig. 10c. We note that the implementation of only screening policy is economically profitable than that of only treatment policy, whereas the combination of screening and treatment is economically most profitable control policy. A small negative profit is observed initially in Fig. 10c which shows that at the beginning none of the control policies are economically profitable. This is because of the sharp growth of infective at the initial phase as well as the cost of control becomes higher than total productivity loss. Thus, combined execution of both interventions is found more effective that minimizes the cost of implemented control policies and disease burden during the outbreak of the epidemic.

\section{Effect of Self-Protection on Optimal Controls}

In this section, we shall explore the effect of self-protection among susceptible individual on the disease dynamics while both controls are implemented. The parameter related to self-protection $(\theta)$ is varied in the range 0 to 0.45 and corresponding populations profiles are plotted in Fig. 11 and corresponding profiles of cost and optimal control functions are plotted in Fig. 12. A higher level of self-protection reduces the cumulative count of infective and also minimizes the duration of peak. From Fig. 12, it is easy to note a higher level of self-protection not only reduces the total cost but also requires a lesser effort of screening and treatment to suppress the disease prevalence. Thus as the level self-protection increases, lower efforts on screening and treatment are sufficient to reduce the level of infection that ultimately minimizes the total incurred cost. Thus, the combination of self-protection along with optimal interventions will be an economically viable option for containing the disease. 


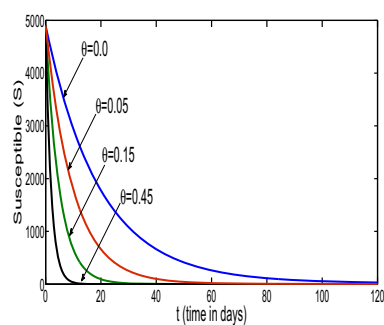

(a)

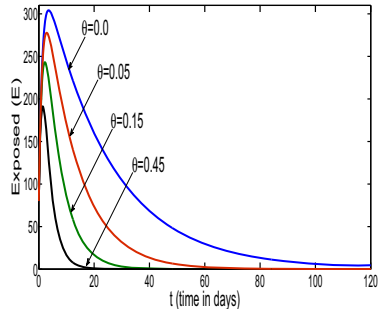

(b)

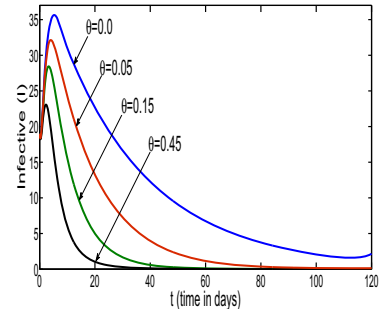

(c)

Fig. 11 a Effect of self-protection $(\theta)$ on $S$ under optimal controls $u_{1}^{*}$ and $u_{2}^{*}$. b Effect of self-protection $(\theta)$ on $E$ under optimal controls $u_{1}^{*}$ and $u_{2}^{*}$. $\mathbf{c}$ Effect of self-protection $(\theta)$ on $I$ under optimal controls $u_{1}^{*}$ and $u_{2}^{*}$

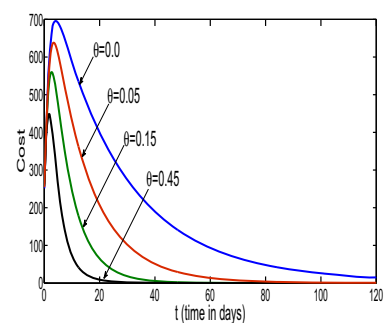

(a)

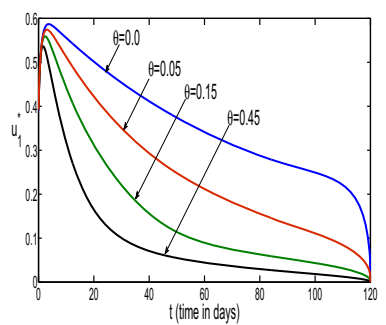

(b)

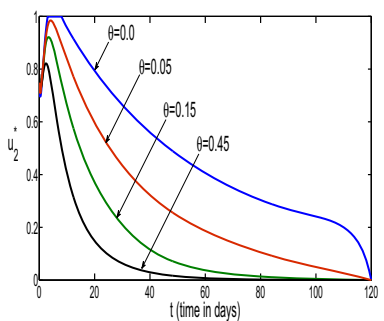

(c)

Fig. 12 a Effect of self-protection $(\theta)$ on cost. b Profiles of optimal control $u_{1}^{*}$ under various values of self-protection $(\theta)$. $\mathbf{c}$ Profiles of optimal control $u_{2}^{*}$ under various values of self-protection $(\theta)$

\section{Effect of Weight Constants on Optimal Controls}

In practice, it is very difficult to get the actual estimates of loss due to disease and costs of applied control interventions. These are related to the weight constants of the optimal control function. Thus, in this section, we analyse the effect of these weight constants on the on the disease as well as on cost and optimal controls. First, we vary the constant $A_{2}$, which is related to opportunity loss, and other weight constants same as earlier. The results plotted in Figs. 13 and 14 show that increasing the weight constant $A_{2}$ requires a higher intensity of applied controls to reduce the infective count. This causes higher cost. No significant change is found in the exposed population as $A_{2}$ increases. It is also clear, from Fig. 14, that lower efforts on optimal controls are enough to reduce the disease burden and economic load when $A_{2}$ is very less.

We further vary the weight constant $B_{1}$ that is involved in the screening process keeping other weight same as earlier. Figure 15 shows significant changes is in the pattern of exposed and infected individuals in this case. Count of infective decreases as $B_{1}$ decreases. Also, from Fig. 16, the optimal screening effort decreases while the optimal treatment increases with increase in $B_{1}$. This increase in optimal treatment causes increment in cost profile (see Fig. 15c).

Finally, we plot the numerical results in Figs. 17 and 18 for the various values of the weight constant $B_{2}$ which is associated with treatment process and related efforts. A similar kind of pattern for infective and the associated cost is found as in the case of constant $B_{1}$. From Fig. 18, we observe that as $B_{2}$ increases, the optimal treatment level reduces while optimal screening level increases. Thus we conclude that the increase in weight constant 


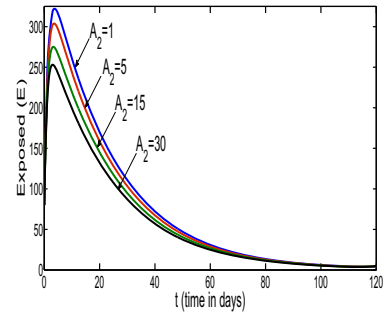

(a)

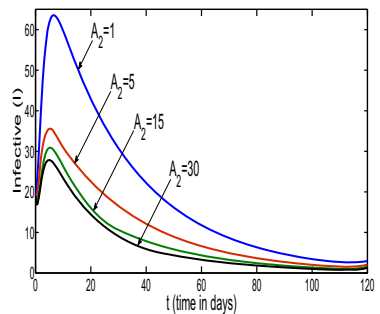

(b)

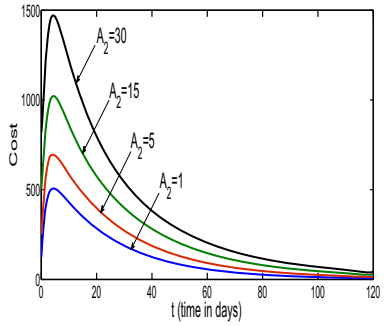

(c)

Fig. 13 a Effect of various $A_{2}$ on exposed individuals. b Effect of various $A_{2}$ on infected individuals. c Effect of various $A_{2}$ on corresponding associated cost. Other weight constants are same as $A_{1}=1, B_{1}=1000$ and $B_{2}=100$

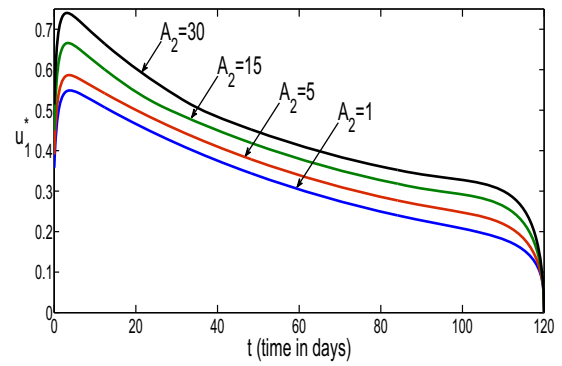

(a)

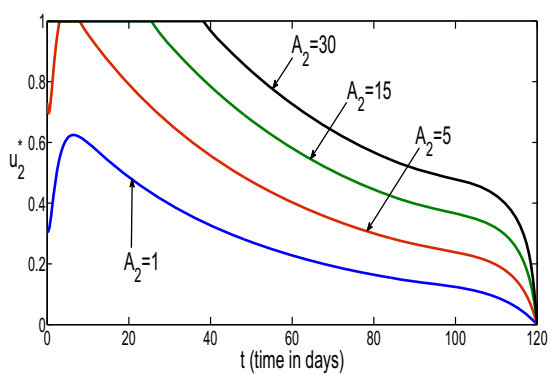

(b)

Fig. 14 a Profiles of optimal control $u_{1}^{*}$ under various $A_{2}$. b Profiles of optimal control $u_{2}^{*}$ under various $A_{2}$. Other weight constants are same as $A_{1}=1, B_{1}=1000$ and $B_{2}=100$

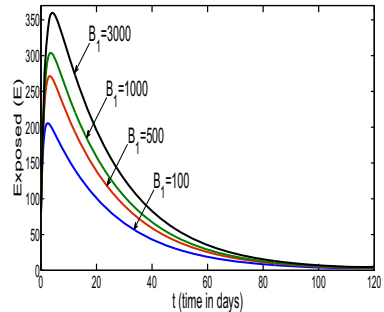

(a)

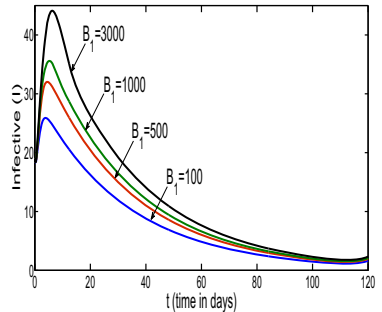

(b)

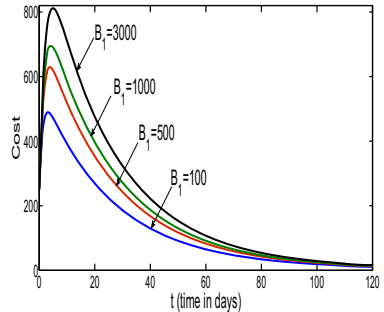

(c)

Fig. 15 a Effect of various $B_{1}$ on exposed individuals. b Effect of various $B_{1}$ on infected individuals. $\mathbf{c}$ Effect of various $B_{1}$ on corresponding associated cost. Other weight constants are same as $A_{1}=1, A_{2}=5$ and $B_{2}=100$

corresponding to a control has an increasing effect on the optimal level of other control and a decreasing effect on the optimal level of that control.

\section{The Effect of $R_{0}$ on the Optimal Control Problem}

In general, it has been noticed that the higher values of the basic reproduction number $R_{0}$ give rise to epidemics characterized by high epidemic peaks [37]. Therefore, in this section, we shall explore how disease burden is affected as $R_{0}$ varies. We shall consider various scenarios 


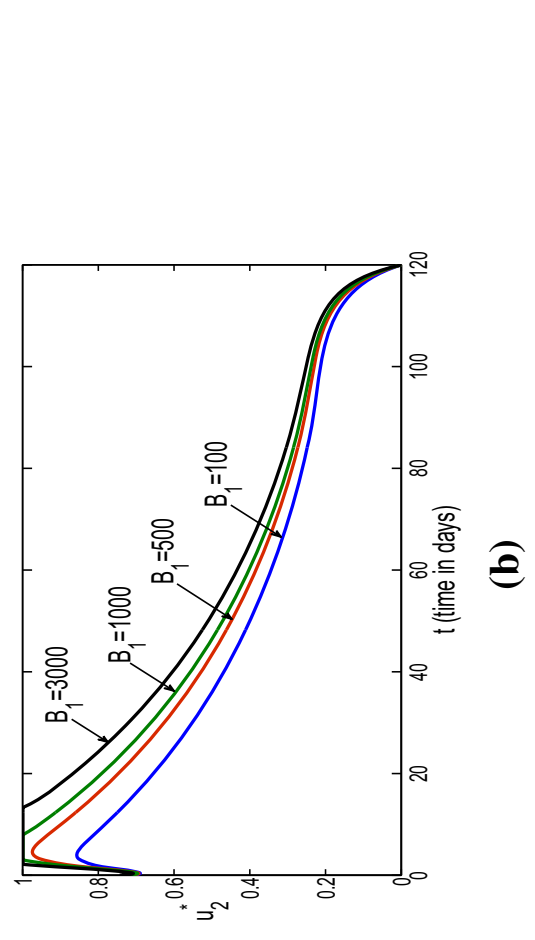

8

I

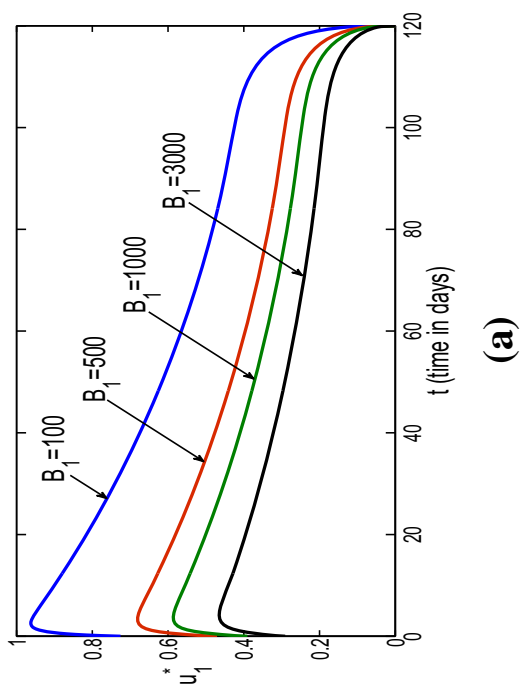

政

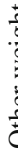

0
0
$\vdots$
0
0
0
$\vdots$
0
0
0
0
0
0
0
0
0
0
0
0
0
0

?ำำ

它

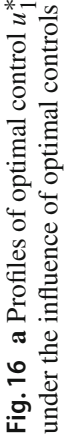




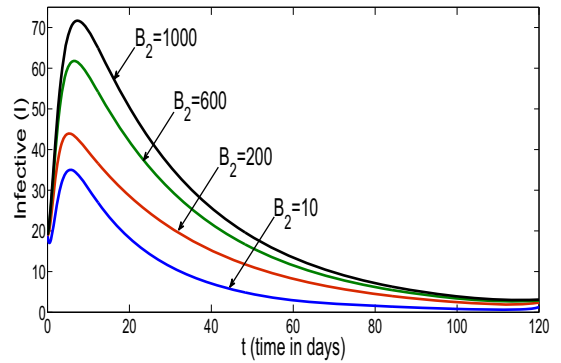

(a)

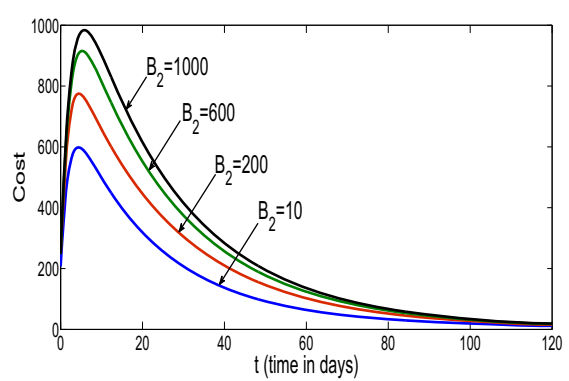

(b)

Fig. 17 a Effect of various $B_{2}$ on infected individuals. b Effect of various $B_{2}$ on corresponding associated cost. Other weight constants are same as $A_{1}=1, A_{2}=5$ and $B_{1}=1000$ under the influence of optimal controls

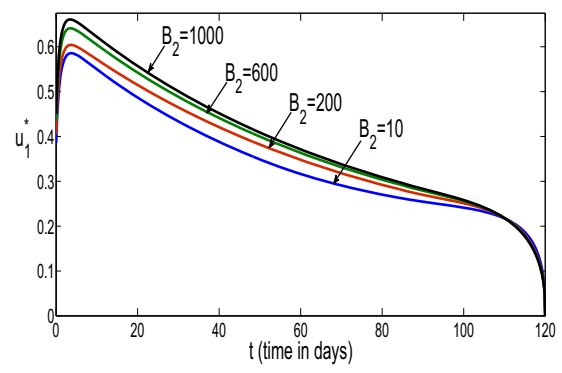

(a)

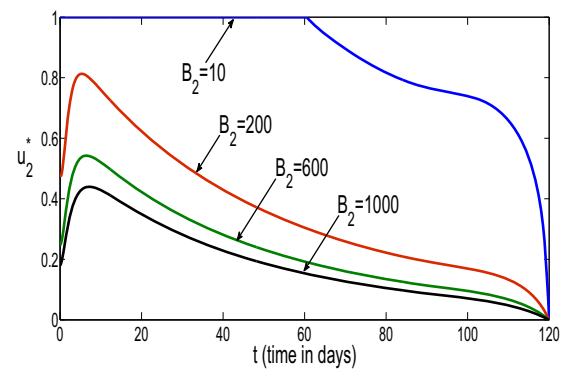

(b)

Fig. 18 a Profiles of optimal control $u_{1}^{*}$ under various $B_{2}$. b Profiles of optimal control $u_{2}^{*}$ under various $B_{2}$. Others weight constants are same as $A_{1}=1, A_{2}=5$ and $B_{1}=1000$ under the influence of optimal controls

of applied control interventions and see how the disease is affected for different values of $R_{0}$. For this purpose, note that the basic reproduction number $R_{0}$ of the system (1), in absence of any controls interventions i.e. $u_{1}=0$ and $u_{2}=0$ is given as:

$$
R_{0}=\frac{\epsilon \beta \Lambda}{(\mu+\theta)(\mu+\epsilon)(\mu+d+\gamma)} .
$$

We choose the parameters same as taken in "Numerical Experimentation with Discussion for the Optimal Control Problem" for making the comparative study numerically. We plot the final epidemic size by varying the degree of transmissibility as reflected by the basic reproduction number $R_{0}$ for different control strategies. The corresponding results are depicted in Fig. 19. It is observed that the combined effect of both the controls $\left(u_{1}^{*}\right.$ and $\left.u_{2}^{*}\right)$ is highly effective in reducing the epidemic load even when the basic reproduction number is very high (approximate $R_{0}=9$ ) as shown in black colored curve in the Fig. 19a. What is noteworthy here is that the effect of only screening $u_{1}^{*}$ in the absence of treatment $\left(u_{2}=0\right)$ also significantly reduces the cumulative disease burden as compared to the case of only treatment (see the red and green colored cures in the Fig. 19a. Also, in the case of only treatment, the count of cumulative disease burden increases for the higher values of the basic reproduction number $R_{0} \in(5,9)$. Hence, the effect of only screening in the absence of treatment plays a vital role in reducing the epidemic burden even for higher values of $R_{0}$. However, no significant effect is seen for the case of combined controls. The corresponding optimal cost is also plotted in 


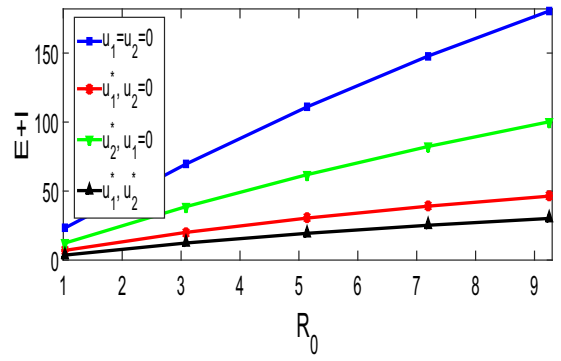

(a)

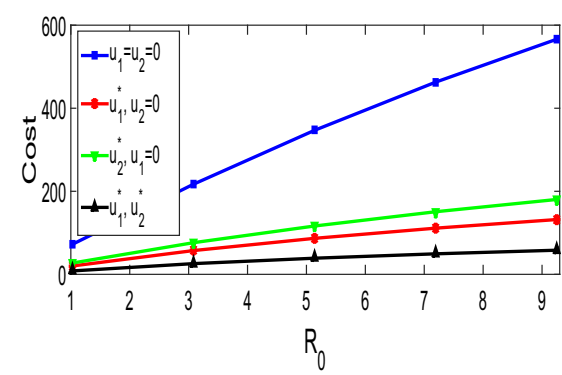

(b)

Fig. 19 Effect of the $R_{0}$ a on the cumulative count of the disease. $\mathbf{b}$ on the corresponding optimal cost

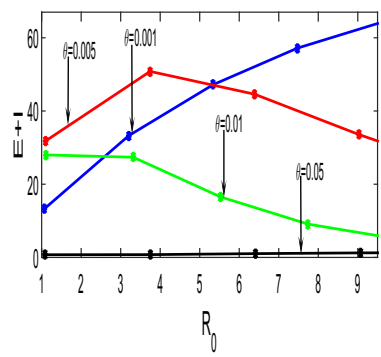

(a)

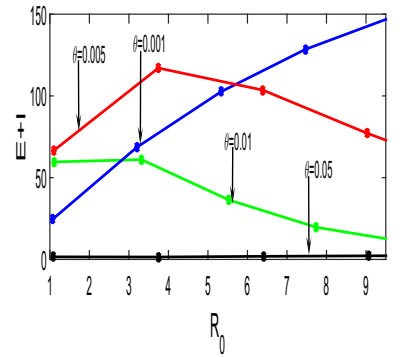

(b)

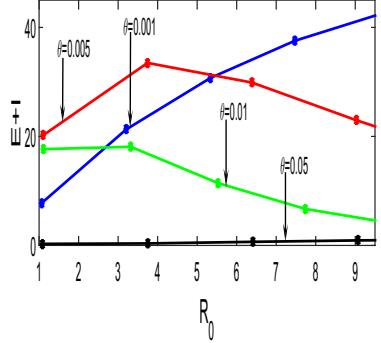

(c)

Fig. 20 Effect of $\theta$ on the cumulative disease burden $\mathbf{a} u_{1}^{*}$ and $u_{2}=0$ (only screening). $\mathbf{b} u_{2}^{*}$ and $u_{1}=0$ (only treatment). $\mathbf{c} u_{1}^{*}$ and $u_{2}^{*}$ (both screening and treatment)

the Fig. 19b. When both the controls are executed together, the cost is also at minimal level for entire range of $R_{0}$.

\section{The Effect of Self-protection $(\theta)$ and $R_{0}$ on the Optimal Controls}

Here, we shall explore the effect of self-protection $\theta$ and $R_{0}$ on the disease dynamics. For, this purpose, we plot the final epidemic size by varying $\theta \in[0.001,0.05]$ for different control strategies and the corresponding results are shown in Fig. 20. Figure 21 gives the corresponding cost distribution in each cases. Our study accentuates that the self-protection due to the information plays a crucial role in reducing the epidemic size even for higher values of $R_{0}$. From Fig. 20a ,b, we found that, the only screening along with high self-protection potentially reduces the epidemic size for all values of $R_{0}$ than the case of only treatment. Whereas, only treatment along with self-protection also affects the disease progression significantly. Moreover, the comprehensive impact of both the optimal controls along with high level self-protection keeps the disease prevalence at a very lower level for all values of $R_{0}$. We also found that the corresponding cost in each case as discussed above is also at lower level (Fig. 21). Thus, the combined effect of self-protection due to information and optimal controls is highly effective and economical viable even if for higher values of $R_{0}$. 


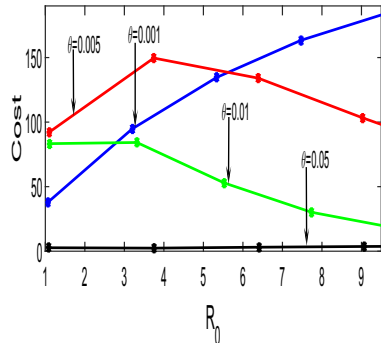

(a)

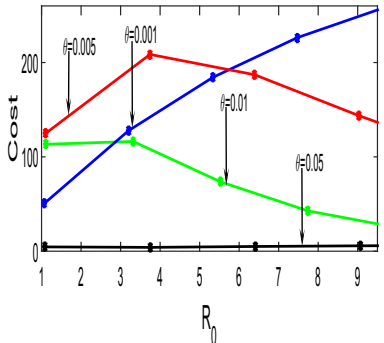

(b)

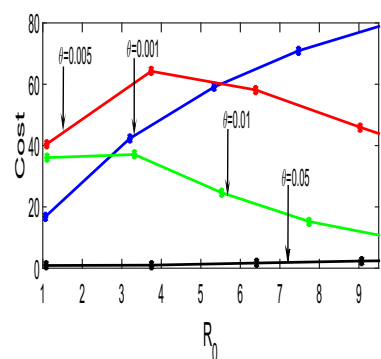

(c)

Fig. 21 Effect of $\theta$ on optimal cost $\mathbf{a} u_{1}^{*}$ and $u_{2}=0$ (only screening). $\mathbf{b} u_{2}^{*}$ and $u_{1}=0$ (only treatment). $\mathbf{c} u_{1}^{*}$ and $u_{2}^{*}$ (both screening and treatment)

\section{Conclusion}

In this work, we proposed and analysed a nonlinear SEIR compartmental model that accounts for the screening of exposed class and saturated treatment on infective. In this model, the effect of self-protection has also been quantified in susceptible population so that susceptible can reduce the chance of infection via protective measures. Model analysis has been performed via equilibrium analysis and found that whenever the basic reproduction number $R_{0}$ is greater than unity there exists a unique globally asymptotic stable endemic equilibrium. Whereas when $R_{0}<1$ under certain conditions, along with the disease free equilibrium two more endemic equilibria exist. And in this case, the saturation in treatment leads to the backward bifurcation. Thus due to limited medical resources, infective stay in population for the case $R_{0}<1$ that generally shows the eradication of infection.

Further, we proposed an optimal control problem by incorporating screening and treatment as control interventions and explored the impact of optimal controls on the reduction of the disease burden and economic load. Total cost construction has been coined that consists the costs associated in the implementation of control interventions and the cost due to disease burden. We found that screening policy on exposed individuals is more effective and less expensive than the treatment only policy on infective. Whereas the comprehensive use of screening and treatment works better than any single control policy and found most effective and less expensive. Also, combined usage of screening and treatment almost eradicated the cumulative count of infection or contained at very low level.

Thus, the combination of screening and treatment is found economically profitable. Also, in between the single control policies of screening only and treatment only, screening is found significantly economically profitable. We also analysed the effect of self-protection on susceptible population and found that as the level self-protection increases, lower efforts on screening and treatment are sufficient to reduce the level of infection that ultimately minimizes the total incurred cost. Numerically we also note that the epidemic peak as well as cost is reduced when self-protection is high for various values of basic reproduction number. Thus, the combination of self-protection along with optimal interventions will be an economically viable option for containing the disease. Also, the effect of positive weight constants on the disease dynamics has also been analysed and found that these constants pay a crucial role in balancing economic burden during the course of infection.

Acknowledgements The work of first author [Anuj Kumar] is financially supported by Council of Scientific and Industrial Research, India (Grant No.: 09/1023(0009)/2012-EMR-I). The authors are thankful to the 
anonymous reviewers for their constructive comments and suggestions which helped in significant improvement of the manuscript.

\section{Appendix A: Global Stability of Endemic Equilibrium Point $E_{2}$}

Theorem 7 If $R_{0}>1$, the model system (2) has unique infected equilibrium point $E_{2}$ which is globally asymptotically stable provided

$$
\left(\epsilon\left(1+\alpha_{1} I_{*}\right)\left(\mu+\alpha_{1} \Lambda\right)+\beta \Lambda\right)^{2}<\frac{4 \beta I_{*} \mu^{2}\left(1+\alpha_{1} I_{*}\right)(\mu+\delta+\gamma)\left(\mu+\epsilon+u_{1}\right)}{\left(2 \mu+\theta+\epsilon+u_{1}\right)} .
$$

Proof Consider the following positive definite function in $\Gamma$,

$$
V(S, E, I)=\frac{m_{1}}{2}\left[\left(S-S_{*}\right)+\left(E-E_{*}\right)\right]^{2}+\frac{1}{2}\left(E-E_{*}\right)^{2}+\frac{1}{2}\left(I-I_{*}\right)^{2} .
$$

Here $m_{1}$ is positive constant and will be chosen later. Differentiating $V$ with respect to $t$ along the solution trajectories of system (2) and using following relations:

$$
\begin{aligned}
(\mu+\theta) S_{*}+\left(\mu+\epsilon+u_{1}\right) E_{*} & =\Lambda, \\
\frac{\beta S_{*} I_{*}}{1+m I}-\left(\mu+\epsilon+u_{1}\right) E_{*} & =0, \\
\epsilon E_{*}-(\mu+d+\gamma) I_{*}-\frac{u_{2} I_{*}}{1+\alpha I_{*}} & =0 .
\end{aligned}
$$

We have,

$$
\begin{aligned}
\dot{V}= & m_{1}\left[\left(S-S_{*}\right)+\left(E-E_{*}\right)\right] \frac{d(S+E)}{d t}+\left(E-E_{*}\right) \frac{d E}{d t}+\left(I-I_{*}\right) \frac{d I}{d t} \\
= & m_{1}\left[\left(S-S_{*}\right)+\left(E-E_{*}\right)\right]\left[\Lambda-(\mu+\theta) S-\left(\mu+\epsilon+u_{1}\right) E\right]+\left(E-E_{*}\right)\left[\frac{\beta S I}{1+m I}\right. \\
& \left.-\left(\mu+\epsilon+u_{1}\right) E\right]+\left(I-I_{*}\right)\left(\epsilon E-(\mu+d+\gamma) I-\frac{u_{2} I}{1+\alpha I}\right) \\
= & m_{1}\left[\left(S-S_{*}\right)+\left(E-E_{*}\right)\right]\left[-(\mu+\theta)\left(S-S_{*}\right)-\left(\mu+\epsilon+u_{1}\right)\left(E-E_{*}\right)\right]+\left(E-E_{*}\right) \\
& {\left[\frac{\beta S\left(I-I_{*}\right)}{(1+m I)\left(1+m I_{*}\right)}+\frac{\beta I_{*}\left(S-S_{*}\right)}{\left(1+m I_{*}\right)}\right]+\left(I-I_{*}\right)\left[\epsilon\left(E-E_{*}\right)-(\mu+d+\gamma)\left(I-I_{*}\right)\right.} \\
& \left.-\frac{u_{2}}{(1+\alpha I)\left(1+\alpha I_{*}\right)}\left(I-I_{*}\right)\right] \\
= & -m_{1}(\mu+\theta)\left(S-S_{*}\right)^{2}-m_{1}\left(2 \mu+\theta+\epsilon+u_{1}\right)\left(S-S_{*}\right)\left(E-E_{*}\right)-m_{1}\left(\mu+\theta+u_{1}\right) \\
& \left(E-E_{*}\right)^{2}+\left(\epsilon+\frac{\beta S}{(1+m I)\left(1+m I_{*}\right)}\right)\left(I-I_{*}\right)\left(E-E_{*}\right)+\frac{\beta I_{*}}{\left(1+m I_{*}\right)}\left(S-S_{*}\right) \\
& \left(E-E_{*}\right)-\left(\mu+d+\gamma+\frac{u_{2}}{(1+\alpha I)\left(1+\alpha I_{*}\right)}\right)\left(I-I_{*}\right)^{2} .
\end{aligned}
$$

Choose $m_{1}=\frac{\beta I_{*}}{\left(1+m I_{*}\right)\left(2 \mu+\theta+\epsilon+u_{1}\right)}$, we get

$$
\begin{aligned}
\dot{V}= & -\frac{\beta I_{*}(\mu+\theta)}{\left(1+m I_{*}\right)\left(2 \mu+\theta+\epsilon+u_{1}\right)}\left(S-S_{*}\right)^{2}-\frac{u_{2}}{(1+\alpha I)\left(1+\alpha I_{*}\right)}\left(I-I_{*}\right)^{2}-\left(E-E_{*}\right)^{2} \\
& \frac{\beta I_{*}\left(\mu+\theta+u_{1}\right)}{\left(1+m I_{*}\right)\left(2 \mu+\theta+\epsilon+u_{1}\right)}+\left(\epsilon+\frac{\beta S}{(1+m I)\left(1+m I_{*}\right)}\right)\left(I-I_{*}\right)\left(E-E_{*}\right)
\end{aligned}
$$




$$
-(\mu+d+\gamma)\left(I-I_{*}\right)^{2} .
$$

Clearly $\dot{V} \leq 0$ in $\Gamma$ provided following condition is satisfied,

$$
\left(\epsilon\left(1+\alpha_{1} I_{*}\right)\left(\mu+\alpha_{1} \Lambda\right)+\beta \Lambda\right)^{2}<\frac{4 \beta I_{*} \mu^{2}\left(1+\alpha_{1} I_{*}\right)(\mu+\delta+\gamma)\left(\mu+\epsilon+u_{1}\right)}{\left(2 \mu+\theta+\epsilon+u_{1}\right)} .
$$

Hence $\dot{V}<0$ and $\dot{V}=0$ if and only if $S=S_{*}, E=E_{*}$ and $I=I_{*}$ in $\Gamma$. Thus the singleton set $\left\{E_{2}\right\}$ is the largest positively invariant set contained in $\{(S, E, I) \in \Gamma: \dot{V}=0\}$. By Lyapunov LaSalle Theorem [34] $E_{2}$ is globally asymptotically stable in the interior of $\Gamma$.

\section{Appendix B: Direction of Bifurcation at $R_{0}=1$}

In this section, we reproduce the method given by Chavez and Song [12] to establish the direction of bifurcation at the crucial threshold $R_{0}=1$.

Theorem 8 [12] Consider the following system of ODEs with a parameter $\phi$ :

$$
\frac{d x}{d t}=f(x, \phi), \quad f: \mathbb{R}^{n} \times \mathbb{R} \rightarrow \mathbb{R}^{n} \text { and } f \in \mathbb{C}^{2}\left(\mathbb{R}^{n} \times \mathbb{R}\right),
$$

with 0 is an equilibrium of this system and $f(0, \phi)=0$ for all $\phi$. Assume

$P_{1}: A=D_{x} f(0,0)=\left(\frac{\partial f_{i}}{\partial x_{j}}(0,0)\right)$ is the linearization matrix of the system (11) around the equilibrium 0 with $\phi$ evaluated at 0 . Zero is a simple eigenvalue of $A$ and all other eigenvalues of A have negative real parts.

$P_{2}$ : Matrix A has a non-negative right eigenvector y and a left eigenvector $z$ corresponding to the zero eigenvalue.

Let $f_{k}$ be the kth component of $f$ and

$$
\begin{aligned}
& a=\sum_{k, i, j=1}^{n} z_{k} y_{i} y_{j} \frac{\partial^{2} f_{k}}{\partial x_{i} \partial x_{j}}(0,0), \\
& b=\sum_{k, i=1}^{n} z_{k} y_{i} \frac{\partial^{2} f_{k}}{\partial x_{i} \partial \phi}(0,0) .
\end{aligned}
$$

The local dynamics of the system (11) around 0 is totally determined by $a$ and $b$.

(i) $a>0, b>0$. When $\phi<0$ with $|\phi| \ll 1,0$ is locally asymptotically stable, and there exists a positive unstable equilibrium; when $0<\phi \ll 1,0$ is unstable and there exists a negative and locally asymptotically stable equilibrium.

(ii) $a<0, b<0$. When $\phi<0$ with $|\phi| \ll 1,0$ is unstable; when $0<\phi \ll 1,0$ is locally asymptotically stable, and there exists a positive unstable equilibrium.

(iii) $a>0, b<0$. When $\phi<0$ with $|\phi| \ll 1,0$ is unstable, and there exists a locally asymptotically stable negative equilibrium; when $0<\phi \ll 1,0$ is stable, and a positive unstable equilibrium appears.

(iv) $a<0, b>0$. When $\phi$ changes from negative to positive, 0 changes its stability from stable to unstable. Corresponding a negative unstable equilibrium becomes positive and locally asymptotically stable. 


\section{Appendix C: Optimality System}

Here, we represent the optimality system using optimal control functions that are characterized in the above section. Optimality system with minimized Hamiltonian $H^{*}$ at $\left(S^{*}, E^{*}, I^{*}, R^{*}, u_{1}^{*}, u_{2}^{*}, \lambda\right)$ is given as:

$$
\begin{aligned}
\frac{d S^{*}}{d t} & =\Lambda-\frac{\beta S^{*} I^{*}}{1+m I^{*}}-\mu S^{*}-\theta S^{*}, \\
\frac{d E^{*}}{d t} & =\frac{\beta S^{*} I^{*}}{1+m I^{*}}-\left(\mu+\epsilon+u_{1}^{*}\right) E^{*}, \\
\frac{d I^{*}}{d t} & =\epsilon E^{*}-(\mu+d+\gamma) I^{*}-\frac{u_{2}^{*} I^{*}}{1+\alpha I^{*}}, \\
\frac{d R^{*}}{d t} & =\gamma I^{*}-\mu R^{*}+u_{1}^{*} E^{*}+\frac{u_{2} I^{*}}{1+\alpha I^{*}}+\theta S^{*},
\end{aligned}
$$

with initial conditions $S^{*}(0)>0, E^{*}(0) \geq 0, I^{*}(0) \geq 0$ and $R^{*}(0) \geq 0$. And the adjoint system is

$$
\begin{aligned}
\frac{d \lambda_{1}}{d t}= & \left(\mu+\theta+\frac{\beta I^{*}}{1+m I^{*}}\right) \lambda_{1}-\frac{\beta I^{*}}{1+m I^{*}} \lambda_{2}-\theta \lambda_{4}, \\
\frac{d \lambda_{2}}{d t}= & -A_{1}+\left(\mu+\epsilon+u_{1}^{*}\right) \lambda_{2}-\epsilon \lambda_{3}-u_{1}^{*} \lambda_{4}, \\
\frac{d \lambda_{3}}{d t}= & -A_{2}+\frac{\beta S^{*}}{\left(1+m I^{*}\right)^{2}} \lambda_{1}-\frac{\beta S^{*}}{\left(1+m I^{*}\right)^{2}} \lambda_{2}+\left(\mu+d+\gamma+\frac{u_{2}^{*}}{\left(1+\alpha I^{*}\right)^{2}}\right) \lambda_{3} \\
& -\left(\gamma+\frac{u_{2}^{*}}{\left(1+\alpha I^{*}\right)^{2}}\right) \lambda_{4}, \\
\frac{d \lambda_{4}}{d t}= & \mu \lambda_{4},
\end{aligned}
$$

with transversality conditions $\lambda_{1}(T)=0, \lambda_{2}(T)=0, \lambda_{3}(T)=0$ and $\lambda_{4}(T)=0$. The optimal control functions $u_{1}^{*}$ and $u_{2}^{*}$ are given as in (9) and (10).

\section{References}

1. CBC News: The economic impact of SARS. CBC News Online, July 2003. http://www.cbc.ca/news2/ 745background/sars/economicimpact.html. Accessed 25 Mar 2019

2. National AIDS Control Organisation, India: Annual report 2010-2011. http://www.naco.gov.in/sites/ default/files/NACO\%20Annual\%20Report\%202010-11.pdf. Accessed 25 Mar 2019

3. The Economic and Social Impact of Emerging Infectious Disease. http://healthcare.philips.com/main/ shared/assets/documents/bioshield/ecoandsocialimpactofemerginginfectiousdisease_111208.pdf. Mar 2015

4. Ahituv, A., Hotz, V.J., Philipson, T.: The responsiveness of the demand for condoms to the local prevalence of AIDS. J. Hum. Resour. 31, 869-897 (1996)

5. Armbruster, B., Brandeau, M.L.: Optimal mix of screening and contact tracing for endemic diseases. Math. Biosci. 209(2), 386-402 (2007)

6. Armbruster, B., Brandeau, M.L.: Cost-effective control of chronic viral diseases: finding the optimal level of screening and contact tracing. Math. Biosci. 224(1), 35-42 (2010)

7. Behncke, H.: Optimal control of deterministic epidemics. Optim. Control Appl. Methods 21(6), 269-285 (2000)

8. Brauer, F., Castillo-Chávez, C.: Mathematical Models in Population Biology and Epidemiology. Springer, Berlin (2012) 
9. Buonomo, B., d'Onofrio, A., Lacitignola, D.: Globally stable endemicity for infectious diseases with information-related changes in contact patterns. Appl. Math. Lett. 25(7), 1056-1060 (2012)

10. Castilho, C.: Optimal control of an epidemic through educational campaigns. Electron. J. Diff. Equ. 2006(125), 1-11 (2006)

11. Castillo-Chavez, C., Feng, Z., Huang, W.: On the computation of $R_{0}$ and its role in global stability. Inst. Math. Appl. 125, 229-250 (2002)

12. Castillo-Chavez, C., Song, B.: Dynamical models of tuberculosis and their applications. Math. Biosci. Eng. 1(2), 361-404 (2004)

13. Coddington, E.A., Levinson, N.: Theory of Ordinary Differential Equations. Tata McGraw-Hill Education, Maidenheach (1955)

14. d'Onofrio, A., Manfredi, P.: Information-related changes in contact patterns may trigger oscillations in the endemic prevalence of infectious diseases. J. Theor. Biol. 256(3), 473-478 (2009)

15. d'Onofrio, A., Manfredi, P., Salinelli, E.: Vaccinating behaviour, information, and the dynamics of SIR vaccine preventable diseases. Theor. Popul. Biol. 71(3), 301-317 (2007)

16. Dubey, B., Patra, A., Srivastava, P.K., Dubey, U.S.: Modeling and analysis of an SEIR model with different types of nonlinear treatment rates. J. Biol. Syst. 21(03), 1320023 (2013)

17. Fleming, W.H., Rishel, R.W.: Deterministic and Stochastic Optimal Control, vol. 1. Springer, New York (1975)

18. Funk, S., Gilad, E., Jansen, V.A.A.: Endemic disease, awareness, and local behavioural response. J. Theor. Biol. 264(2), 501-509 (2010)

19. Gaff, H., Schaefer, E.: Optimal control applied to vaccination and treatment strategies for various epidemiological models. Math. Biosci. Eng. 6(3), 469-492 (2009)

20. Gaff, H.D., Schaefer, E., Lenhart, S.: Use of optimal control models to predict treatment time for managing tick-borne disease. J. Biol. Dyn. 5(5), 517-530 (2011)

21. Grass, D.: Optimal Control of Nonlinear Processes: With Applications in Drugs, Corruption and Terror. Springer, Berlin (2008)

22. Greenhalgh, D., Rana, S., Samanta, S., Sardar, T., Bhattacharya, S., Chattopadhyay, J.: Awareness programs control infectious disease-multiple delay induced mathematical model. Appl. Math. Comput. 251, 539-563 (2015)

23. Gumel, A.B., Ruan, S., et al.: Modelling strategies for controlling SARS outbreaks. Proc. R. Soc. Lond. Ser. B: Biol. Sci. 271(1554), 2223-2232 (2004)

24. Gupta, A.G., Moyer, C.A., Stern, D.T.: The economic impact of quarantine: SARS in Toronto as a case study. J. Infect. 50(5), 386-393 (2005)

25. Hethcote, H.W.: The mathematics of infectious diseases. SIAM Rev. 42(4), 599-653 (2000)

26. Joshi, H.R.: Optimal control of an HIV immunology model. Optim. Control Appl. Methods 23(4), 199213 (2002)

27. Joshi, H.R., Lenhart, S., Li, M.Y., Wang, L.: Optimal control methods applied to disease models. Contemp. Math. 410, 187-208 (2006)

28. Kassa, S.M., Ouhinou, A.: The impact of self-protective measures in the optimal interventions for controlling infectious diseases of human population. J. Math. Biol. 70(1-2), 213-236 (2015)

29. Kim, M.Y.: Existence of steady state solutions to an epidemic model with screening and their asymptotic stability. Appl. Math. Comput. 74(1), 37-58 (1996)

30. Kim, M.Y., Milner, F.A.: A mathematical model of epidemics with screening and variable infectivity. Math. Comput. Model. 21(7), 29-42 (1995)

31. Kirschner, D., Lenhart, S., Serbin, S.: Optimal control of the chemotherapy of HIV. J. Math. Biol. 35(7), 775-792 (1997)

32. Kumar, A., Srivastava, P.K.: Vaccination and treatment as control interventions in an infectious disease model with their cost optimization. Commun. Nonlinear Sci. Numer. Simul. 44, 334-343 (2017)

33. Kumar, A., Srivastava, P.K., Takeuchi, Y.: Modeling the role of information and limited optimal treatment on disease prevalence. J. Theor. Biol. 414, 103-119 (2017)

34. LaSalle, J.P.: The Stability of Dynamical Systems, Regional Conference Series in Applied Mathematics. SIAM, Philadelphia (1976)

35. Lawrence, P.: Differential equations and dynamical systems, vol. 7. Springer-Verlag, New York (1991)

36. Lee, H.L., Pierskalla, W.P., Rand, G.K.: Theory and general models of mass screening for contagious and non-contagious diseases. Oper. Res. 87, 428-441 (1988)

37. Lee, S., Chowell, G., Castillo-Chávez, C.: Optimal control for pandemic influenza: the role of limited antiviral treatment and isolation. J. Theor. Biol. 265(2), 136-150 (2010)

38. Lenhart, S.M., Workman, J.T.: Optimal Control Applied to Biological Models, vol. 15. CRC Press, Bocca Raton (2007) 
39. Liu, X., Takeuchi, Y., Iwami, S.: SVIR epidemic models with vaccination strategies. J. Theor. Biol. 253(1), 1-11 (2008)

40. Liu, Y., Cui, J.: The impact of media coverage on the dynamics of infectious disease. Int. J. Biomath. 1(1), 65-74 (2008)

41. Manfredi, P., d'Onofrio, A.: Modeling the Interplay Between Human Behavior and the Spread of Infectious Diseases. Springer, Berlin (2013)

42. Misra, A.K., Sharma, A., Shukla, J.B.: Modeling and analysis of effects of awareness programs by media on the spread of infectious diseases. Math. Comput. Model. 53(5), 1221-1228 (2011)

43. Moulay, D., Aziz-Alaoui, M.A., Kwon, H.D.: Optimal control of chikungunya disease: larvae reduction, treatment and prevention. Math. Biosci. Eng. 9(2), 369-392 (2012)

44. Okosun, K.O., Makinde, O.D., Takaidza, I.: Impact of optimal control on the treatment of HIV/AIDS and screening of unaware infectives. Appl. Math. Model. 37(6), 3802-3820 (2013)

45. Philipson, T.: Private vaccination and public health: an empirical examination for US measles. J. Hum. Resour. 31, 611-630 (1996)

46. Van den Driessche, P., Watmough, J.: Reproduction numbers and sub-threshold endemic equilibria for compartmental models of disease transmission. Math. Biosci. 180(1), 29-48 (2002)

47. Xiao, D., Ruan, S.: Global analysis of an epidemic model with nonmonotone incidence rate. Math. Biosci. 208(2), 419-429 (2007)

48. Yadav, A., Srivastava, P.K., Kumar, A.: Mathematical model for smoking: effect of determination and education. Int. J. Biomath. 8(01), 1550001 (2015). (14 pages)

49. Yan, X., Zou, Y.: Optimal and sub-optimal quarantine and isolation control in SARS epidemics. Math. Comput. Model. 47(1), 235-245 (2008)

50. Zeiler, I., Caulkins, J.P., Grass, D., Tragler, G.: Keeping options open: an optimal control model with trajectories that reach a dnss point in positive time. SIAM J. Control Optim. 48(6), 3698-3707 (2010)

51. Zhang, J., Jia, J., Song, X.: Analysis of an SEIR epidemic model with saturated incidence and saturated treatment function. Sci. World J. 2014, 1-11 (2014)

52. Zhang, X., Liu, X.: Backward bifurcation and global dynamics of an SIS epidemic model with general incidence rate and treatment. Nonlinear Anal.: Real World Appl. 10(2), 565-575 (2009)

53. Zhou, L., Fan, M.: Dynamics of an SIR epidemic model with limited medical resources revisited. Nonlinear Anal.: Real World Appl. 13(1), 312-324 (2012)

Publisher's Note Springer Nature remains neutral with regard to jurisdictional claims in published maps and institutional affiliations. 